$$
\because \because: \because \text { ISSN26890852 }
$$
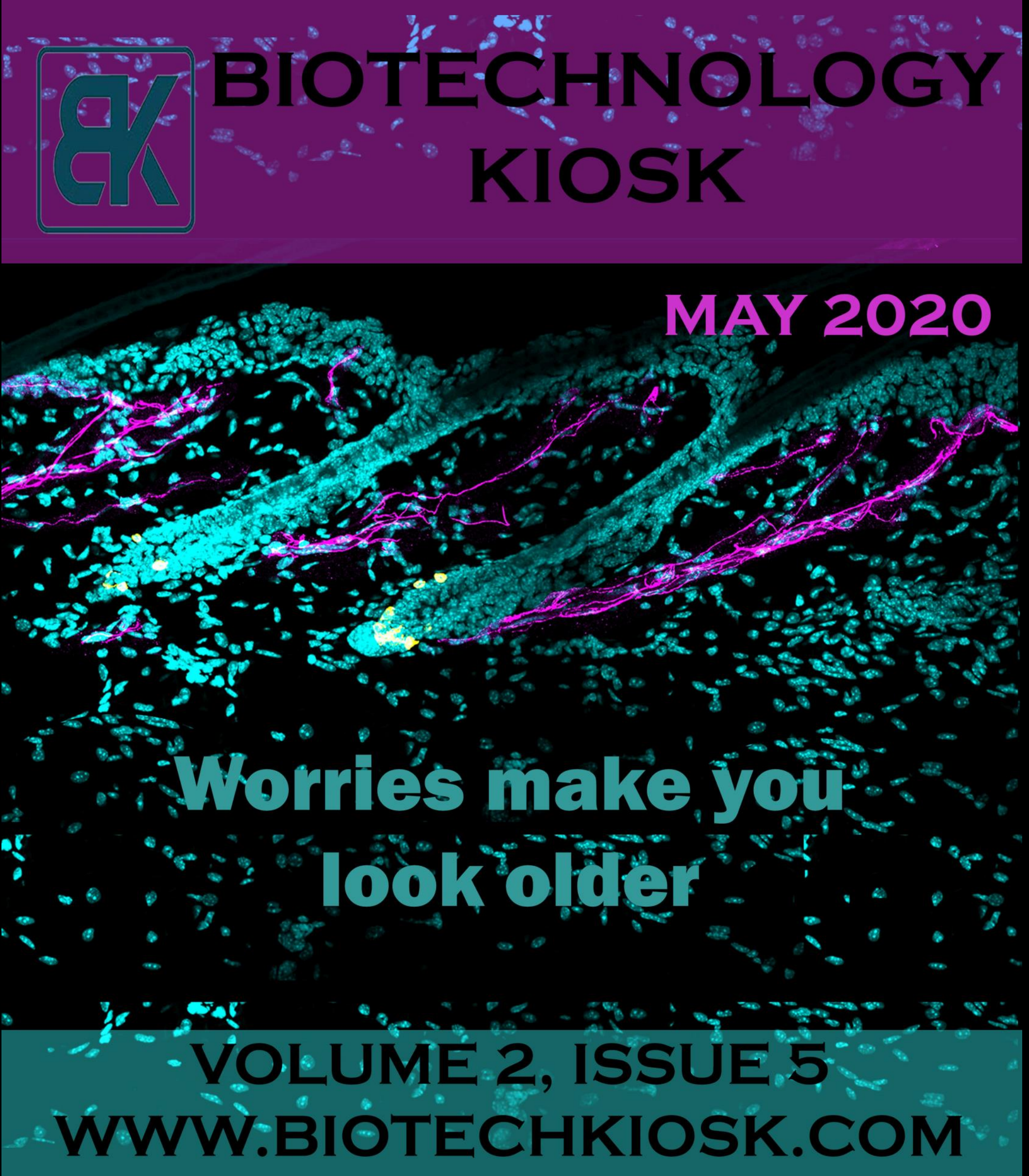

\title{
MAY 2020
}




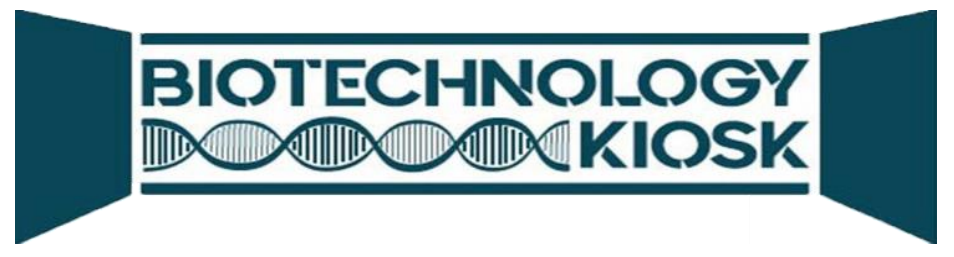

\section{Executive Publishers}

Megha Agrawal, PhD

(Biotechnology)

Publisher and Editor

Expertise:

Neuroscience, Stroke, Pharmacology, Toxicology, Microbiology and Molecular Biology

Email: megha@biotechkiosk.com meghaagra@gmail.com
Shyamasri Biswas, PhD

(Biotechnology)

Publisher and Editor

Expertise:

Structural Biology, Enzyme Technology, and Protein Crystallography

Email: shyabiswas@biotechkiosk.com shyabiswas@gmail.com

\section{Editorial, Sales \& Circulation Office}

160 International Parkway

Suite 100-9, Heathrow

FL-32746, USA

Phone: 386-518-9411

Email: publisher@biotechkiosk.com

www.biotechkiosk.com

ISSN 2689-0852

One stop shop for all things biotech

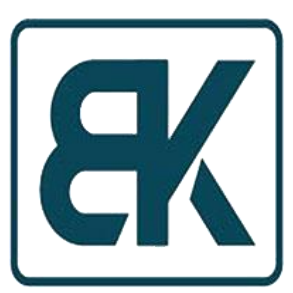




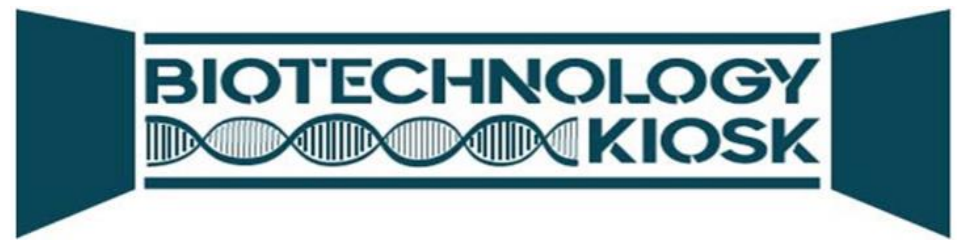

\section{From the Publisher's Desk}

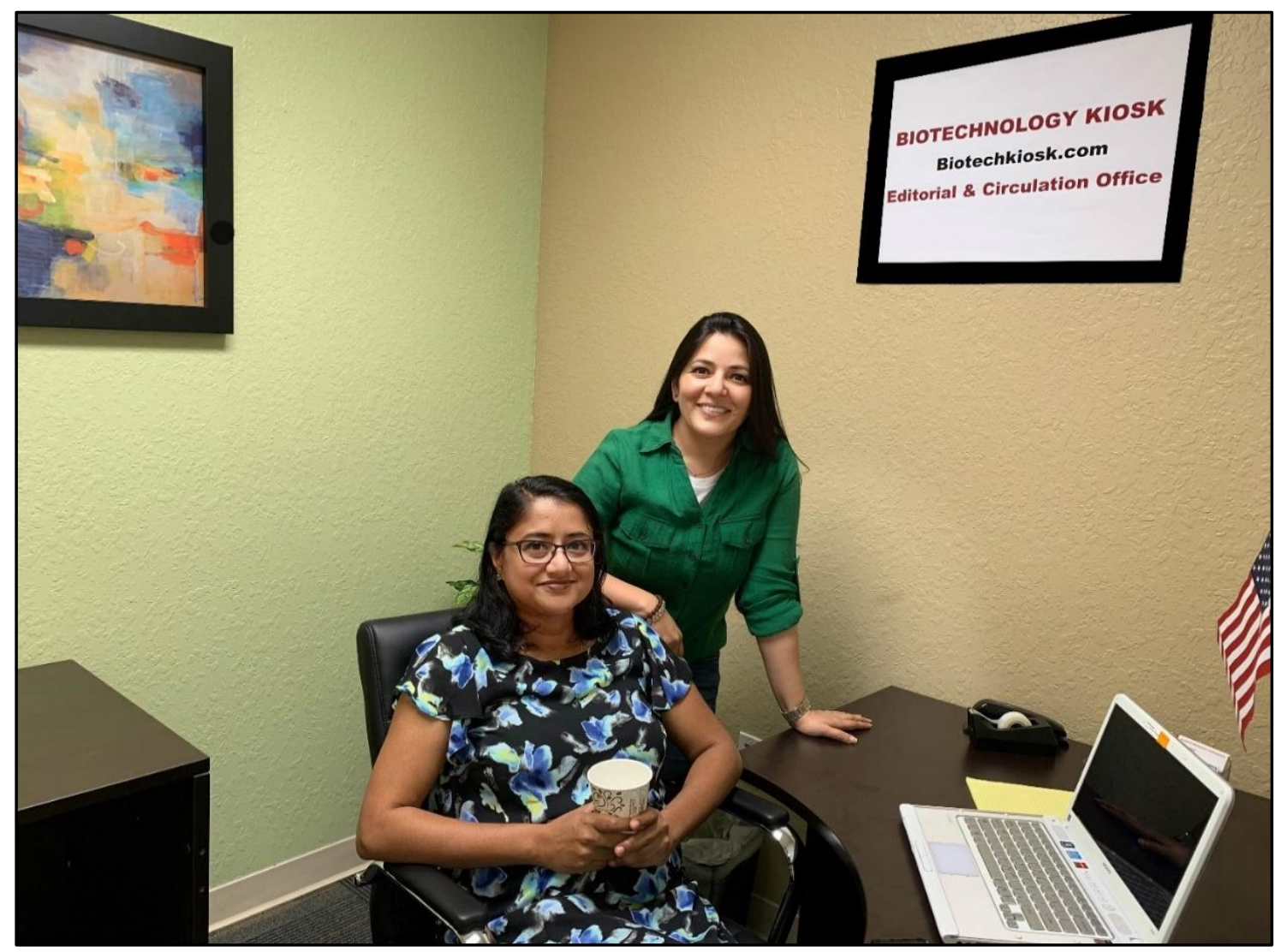

\section{Welcome to Biotechnology Kiosk!}

This issue of Biotechnology Kiosk (BK) is presented to our readers with the regular features that include high-end editorials by experts, biotechnology advances around the world and industry news from pharma and biotech sectors.

This issue contains a number of scholarly editorials, reviews and news and views on the current cutting edge topics that include new findings about positive effects of intermittent fasting on human health, protein engineering in vaccine and therapeutics development, synergistic effects of COVID-
19 and various co-morbidities such as cardiovascular conditions and stress induced medical conditions along with several other topics that have been discussed in the editor's choice that reports on research breakthroughs from around the world.

In view of the COVID-19 pandemic, we have extended the deadline for manuscript submission for the special edition of Vacuum Advances in Biotechnology until July 31,2020 . Please check out the call for papers that is posted on BK's website. We are also now open to consider manuscripts in 
all areas of biotechnology for regular publication in BK. Please go to the sections 'Aims and Scope' to submit your manuscripts to BK.

We look forward to receiving your contributions. We do hope that you will enjoy reading this issue of Biotechnology Kiosk.
Please do write to us with your comments and feedback. Your suggestions are always appreciated.

Dr. Megha Agrawal and Dr. Shyamasri Biswas

Co Editors-in-Chief, Biotechnology Kiosk 


\section{Contents}

Volume 2, Issue 5

May 2020

COLUMNS

PROTEOMICS, DIGESTIVE DISEASES \& METABOLISM 5

Can Intermittent Fasting Protect against Cancer, Metabolic Syndrome, Cognitive and Neuropsychiatric Diseases?

https://doi.org/10.37756/bk.20.2.5.1

PROTEIN ENGINEERING

Designer Proteins for New Vaccines and Therapeutics

https://doi.org/10.37756/bk.20.2.5.2

REVIEW ARTICLES

AGING

Worries make you look older: unraveling the science that connects stress with graying in human beings

https://doi.org/10.37756/bk.20.2.5.3

MYOCARDIAL COMPLICATIONS AND COVID-19 INFECTIONS

Impact of COVID-19 on cardiovascular patients

https://doi.org/10.37756/bk.20.2.5.4

BIOTECH R\&D AND INNOVATION NEWS.

Editor's PICKS: Biotechnology Advances around the World

Cancer Biology

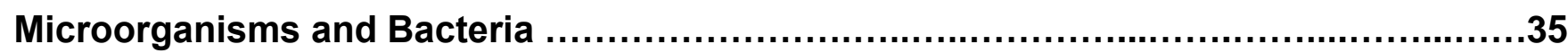

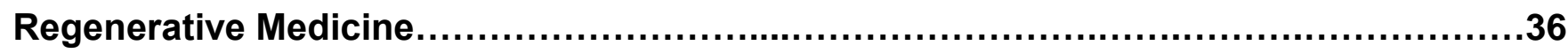

Plant and Agriculture Biotechnology. 
Eli Lilly's Tauvid imaging agent approved by FDA to diagnose AD .38

ZILXI $^{\mathrm{TM}}$ (minocycline), receives FDA approval for rosacea in adults .38

Eagle Pharma to test Ryanodex against COVID-19 38

Meningococcal Vaccines expected to touch USD 9 billion by 2026 39

Novartis partners with Eye \& Ear hospital to manufacture coronavirus vaccine .39

AstraZeneca to study leukemia drug as COVID-19 treatment. 39

Large scale phase $2 \& 3$ trials are underway for coronavirus vaccine. .39

Investments to counter Cancer genes............................................................

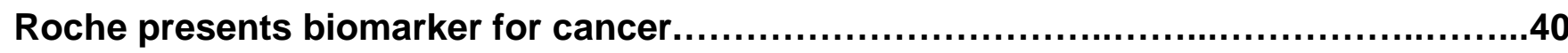

HALIX B.V. to produce vaccines against COVID-19...........................................

SARS-CoV2 antibody tests might not be suitable for individual diagnosis .40 


\title{
COLUMN
}

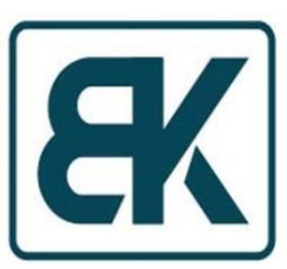

\section{Proteomics, Digestive Diseases \& Metabolism}

By Megha Agrawal*, PhD

Co Editor-in-Chief

Biotechnology Kiosk, 2, 5 (2020)

DOI: https://doi.org/10.37756/bk.20.2.5.1

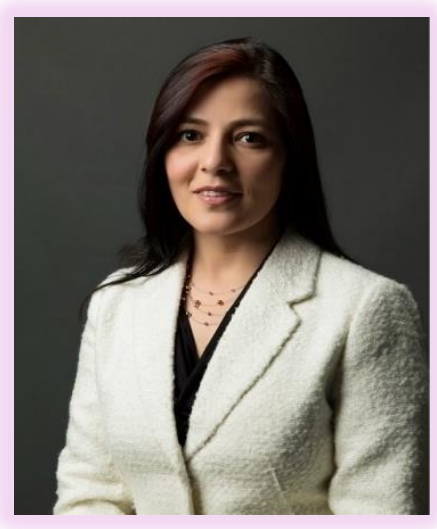

\section{Can Intermittent Fasting Protect against Cancer, Metabolic Syndrome, Cognitive and Neuropsychiatric Diseases?}

\begin{abstract}
Lately, there has been a significant amount of research focus on circadian clock and its impact on human health. It has been shown that disruption of circadian rhythm could lead to cancer and metabolic syndrome. Researchers have identified in a number of murine studies the benefits of time-restricted feeding that can reset the disrupted clock rhythm, which can subsequently optimize the functioning of critical regulatory proteins of metabolism in mice. This has prompted a huge research interest in studying time-restricted feeding in humans for protective actions against cancer and metabolic syndrome and many other complex medical conditions. To this end, a hypothesis based on intermittent fasting for several consecutive days without calorie restriction in humans has been recently clinically tested that has shown an induced anticarcinogenic proteome and the key regulatory proteins of glucose and lipid metabolism for a number of significant health benefits. Here, we present a brief overview on circadian rhythm and its disruption and also the effects of time-restricted feeding to reset the detrimental disruption. We describe recent clinical studies showing potential benefits of intermittent fasting as an adjunct therapy in a number complex medical conditions such as cancer, metabolic syndrome, and several cognitive and neuropsychiatric diseases.
\end{abstract}

\section{*Email: megha@biotechkiosk.com}

To cite this article: Agrawal M; Can Intermittent Fasting Protect against Cancer, Metabolic Syndrome, Cognitive and Neuropsychiatric Disease, Biotechnology Kiosk, Vol 2, Issue 5, PP: 5-10 (2020); DOI: https://doi.org/10.37756/bk.20.2.5.1 


\section{Introduction:}

\section{Disruption in Circadian Rhythm and Health Consequences}

Circadian rhythms are found in most living things. They correspond to physical, mental, and behavioral changes that follow a daily cycle. Studies have shown that circadian rhythm among mammals is controlled by an organized system comprising both central and peripheral oscillators that communicate via neural connections and hormonal cues [1]. Researchers have shown that the ability to adjust physiology and behavior in response to daily changes in the environment is critical for survival. Mammals achieve this ability via a master circadian clock in the suprachiasmatic nucleus (SCN) whose role is to coordinate rhythms in cells and tissue function. This occurs throughout the body according to predictable daily variations in individuals [2]. Further, the oscillators are known to be located within individual neurons in the SCN, where approximately 20,000 neurons are found within bilateral SCN. Also, there are peripheral clocks in addition to the master clock in the SCN that are found in a number of vital organs that include the liver, adipose tissue, kidneys, pancreas, and heart $[1,3]$.

Figure 1 shows schematically the core of the mammalian circadian clock that comprises a transcription-translation feedback loop involving the Period (Per1-3) and Cryptochrome genes (Cry1-2) [1]. It shows a heterodimer of transcription factors including clock and also BMAL1 drive the transcription of these target genes, which contain an E-box enhancer element (Figure 1). This results in PER and CRY proteins that subsequently inhibit the clock (BMAL1) complex. In addition to PER and CRY genes, it is shown that the clock (BMAL1) complex also drives the transcription of metabolic genes such as PPAR $\alpha$, Ror $\alpha / \beta$ (Ror), and Rev-Erb $\alpha$ (Rev) (Figure 1) [1].

Researchers have studied the circadian rhythm that have shown that it helps the body anticipate environmental cues that is needed to optimize energy utilization for the diurnal cycles of rest-activity and feeding-fasting [3]. Further, murine studies have shown that disruption of circadian clock rhythmicity could lead to cancer and metabolic syndrome [4].

The reasons for the serious health consequences as a result of the disruption of circadian rhythm have been shown to be associated with alterations in glucose and lipid metabolism and immune system responses, and also carcinogenesis [3]. It has been suggested that adoption of a strategy based on resetting the disrupted rhythm of the circadian clock could help prevent metabolic syndrome, immune system dysfunction, and cancer [3].

Regarding primary mechanisms that govern the resetting of the circadian clock, one dominant mechanism has been proposed that functions through the master clock located in the SCN of the anterior hypothalamus. This works by dark-light cycles of the day. Subsequently, in this process, all peripheral clocks get synchronized by the master clock via neuronal and humoral signals. This process then results in resetting all peripheral clocks, including hepatic clock during ad libitum food consumption. The other mechanism to reset 
the circadian clock is considered a secondary mechanism, which is based on the response to mealtime during rhythmic, consecutive and time-restricted feeding-fasting cycles $[3,5]$.

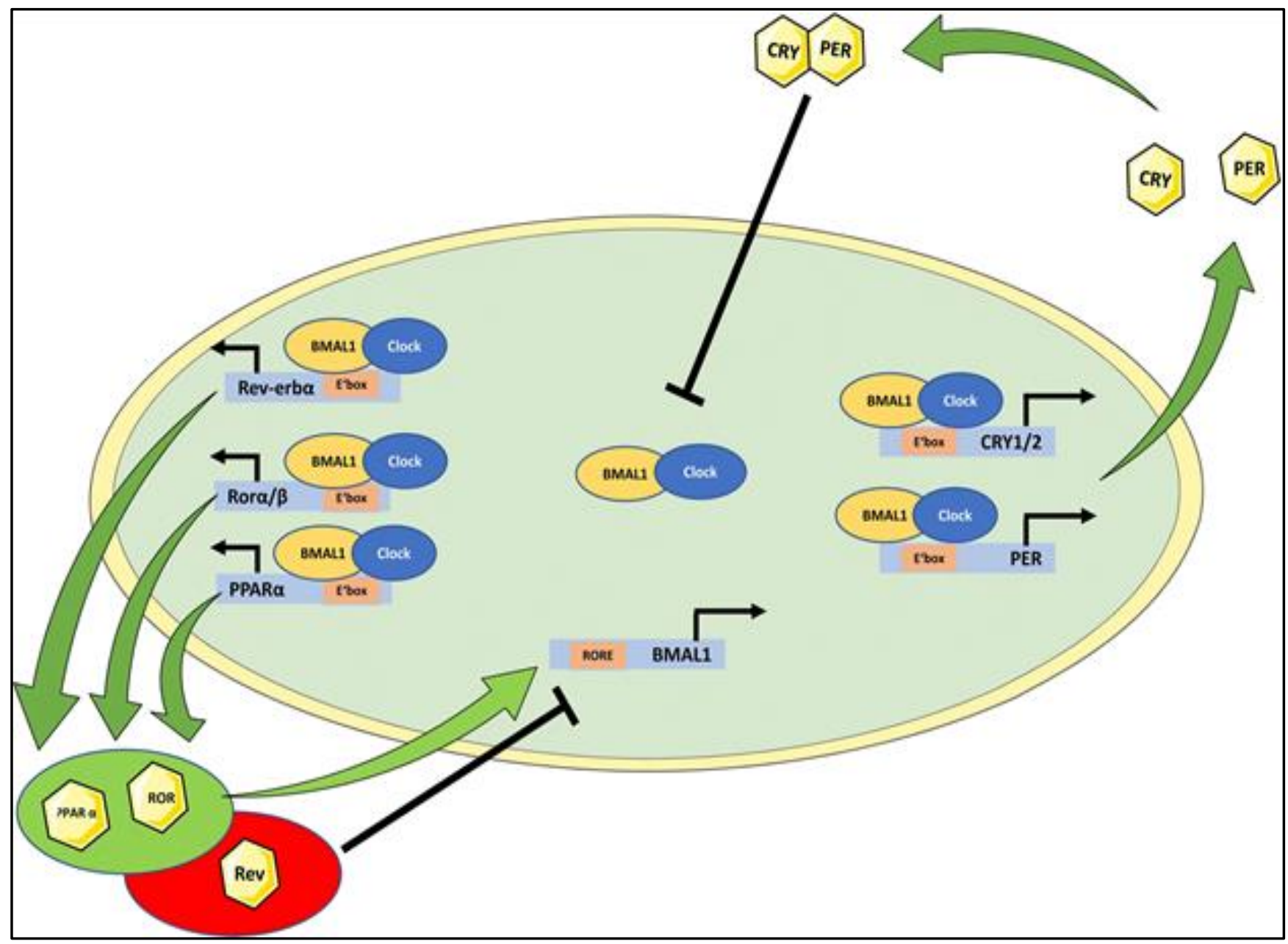

Figure 1: Schematic depiction of a circadian clock machinery showing the core of the circadian clock, which is encoded by transcription-translation feedback loop regulating period (PER) and cryptochrome (CRY) genes [Source: Digestive Diseases and Sciences (2018)].

\section{Time-Restricted Feeding to Reset Disrupted Clock in Humans}

Studies have shown the potential of timerestricted feeding to reset the disrupted clock rhythm that could protect against cancer and metabolic syndrome. In this regard, several murine animal model studies have been carried out that have shown the positive effects of time-restricted access or no access to food during night time/dark phase on resetting the phase of the hepatic clock. This has been shown to optimize the amplitude of hepatic clock oscillations. This subsequently results in the upregulation of mRNA and various protein synthetic pathways especially including enzymes that can play a crucial role in carbohydrate and lipid metabolism [3]. However, the murine animal studies 
employing mice could not address the potentials of time-restricted feeding occurring during the daytime. This is due to the reason that mice are nocturnal feeders, and they consume food mostly at night. On the other hand, in the case of humans, meal intake and most activity usually occur during the daytime [6].

It is of huge clinical significance to study the potentials of time-restricted feeding in humans that allows to reproduce similar optimization in key metabolic regulatory proteins in humans. However, it requires that fasting should occur during the daytime activity for several consecutive days [3]. Further, in such a study, it is of major importance to preserve daytime activity and also timing of the major food consumption at transition zones of the day. In addition, a predawn breakfast and dinner at sunset may be also considered in the time-restricted feeding study due to the significance of caloric content and composition of the food in the prevention of metabolic syndrome and its complications and cancer $[3,6]$.

We present in the following section recent clinical results on intermittent fasting from dawn to sunset that have shown several health benefits including prevention of cancer, metabolic syndrome and Alzheimer's disease and several neuropsychiatric diseases.

\section{Human Study of Serum Proteomics: Important Clinical Implications and Health Benefits}

Researchers conducted the first human study of serum proteomics that included a 30 day dawn-to-sunset intermittent fasting (Figure 2). This study also simultaneously included assessment of clinical metabolic parameters, multiple serum biomarkers, and fecal microbiota in 14 healthy subjects [3].

This study was based on the hypothesis that intermittent fasting for several consecutive days without calorie restriction in humans would induce an anti-carcinogenic proteome and the key regulatory proteins of glucose and lipid metabolism [3]. It involved fourteen healthy subjects that were fasted from dawn to sunset for over $14 \mathrm{~h}$ daily and the fasting duration was continued for 30 consecutive days [3]. Researchers collected serum samples before 30-day intermittent fasting, which occurred at the end of 4 th week during 30-day intermittent fasting, and one week after 30-day intermittent fasting [3]. Subsequently, they performed an untargeted serum proteomic profiling using ultra highperformance liquid chromatography/tandem mass spectrometry. Researchers demonstrated that 30-day intermittent fasting was associated with an anticancer serum proteomic signature. Also, upregulated key regulatory proteins of glucose and lipid metabolism was shown along with circadian clock, DNA repair, cytoskeleton remodeling, immune system, and cognitive function. This resulted in a serum proteome protective against cancer, metabolic syndrome, inflammation, Alzheimer's disease, and several neuropsychiatric disorders [3].

These findings suggested the major clinical significance and positive impact on health as a result of fasting from dawn to sunset for 30 consecutive days. Especially, it is noteworthy that these findings were obtained in the absence of any calorie restriction and significant weight loss [3]. Researchers envisioned that such a time- 
restrictive feeding strategy could be employed in humans as adjunct therapeutic measures for the prevention of cancer,

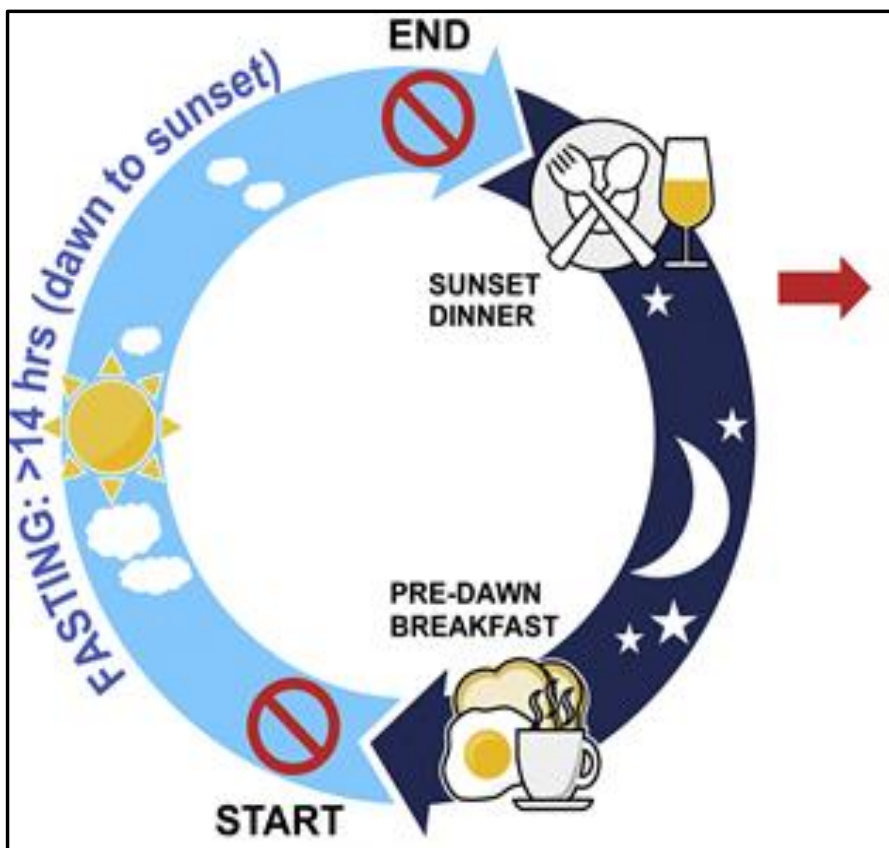

obesity, diabetes, metabolic syndrome, and several cognitive and neuropsychiatric diseases (Figure 2) [3].

\section{Serum Proteome Analysis}

\section{Upregulated Proteomic Signatures}
$\uparrow$ Anticancer proteome
$\uparrow$ Circadian clock
$\uparrow$ DNA repair
$\uparrow$ Insulin signaling
$\uparrow$ Cytoskeleton remodeling
$\uparrow$ Immune system
$\uparrow$ Glucose and lipid metabolism
$\uparrow$ Cognitive function

Figure 2: Schematic presentation of time-restrictive feeding strategy and the health benefits in humans [Source: Journal of Proteomics (2020)].

\section{Conclusion and Outlook}

Research has revealed that the alterations in glucose and lipid metabolism and immune system responses, and carcinogenesis are associated with the disruption of circadian rhythm. Important clinical studies based on intermittent fasting from dawn to sunset have shown novel therapeutic pathways in the prevention of metabolic syndrome, immune system dysfunction, and cancer by resetting the disrupted rhythm of the circadian clock. This innovative therapeutic approach offers promise in the prevention of cancer as well as in several metabolic, inflammatory and immune diseases, Alzheimer's disease and also neuropsychiatric disorders. Recent research has shown that such a strategy based on time-restricted feeding in humans can potentially trigger the important proteome that is protective against carcinogenesis, obesity, diabetes, metabolic syndrome, inflammation, cognitive dysfunction, and mental health.

The existing research data shed light on the functionality of the circadian clock system and metabolic homeostasis. These were shown to be tightly linked and characterized by an extensive crosstalk. However, existing data do not give any clue on the mechanisms of this interaction. These mechanisms are still not understood clearly due to the fact that both aspects of physiology are linked through a complex array of functions. Further research into this 
fundamentally important area could be undertaken. Future research could also focus on targeting the circadian clockcontrolled genes or nuclear receptors, which affect metabolic pathways. This would be useful to pave the way for new therapies in metabolic syndromes and related diseases.

\section{References}

1. Akshay Shetty, Jennifer W. Hsu, Paul P. Manka, Wing-Kin Syn, Role of the Circadian Clock in the Metabolic Syndrome and Nonalcoholic Fatty Liver Disease, Digestive Diseases and Sciences 63:3187-3206 (2018), DOI: https://doi.org/10.1007/s10620-018-5242$\underline{x}$.

2. Paul, S., Hanna, L., Harding, C. et al. Output from VIP cells of the mammalian central clock regulates daily physiological rhythms, Nat Commun 11, 1453 (2020), DOI: https://doi.org/10.1038/s41467-02015277-x.

3. Ayse L. Mindikoglu, Mustafa M. Abdulsada, Antrix Jain Jong Min Choi Prasun K. Jalal Sridevi Devaraj Melissa P.Mezzari Joseph F.Petrosino, Antone R. Opekun, Sung YunJung, Intermittent fasting from dawn to sunset for 30 consecutive days is associated with anticancer proteomic signature and upregulates key regulatory proteins of glucose and lipid metabolism, circadian clock, DNA repair, cytoskeleton remodeling, immune system and cognitive function in healthy subjects, Journal of Proteomics, 217, 103645 (2020), DOI: https://doi.org/10.1016/i.jprot.2020.103645

4. Michael W. Greene, Circadian rhythms and tumor growth, Cancer Letters, 318, 2, 115123 (2012),

DOI: https://doi.org/10.1016/i.canlet.2012.01.00 1 .

5. J.A. Evans, Collective timekeeping among cells of the master circadian clock, J. Endocrinol., 230 (1), R27-R49 (2016), DOI: 10.1530/JOE-16-0054.

6. A.L. Mindikoglu, et al., Impact of timerestricted feeding and dawn-to-sunset fasting on circadian rhythm, obesity, metabolic syndrome, and nonalcoholic fatty liver disease, Gastroenterol. Res. Pract., $3932491 \quad$ (2017), DOI: https://doi.org/10.1155/2017/3932491. 


\title{
COLUMN
}

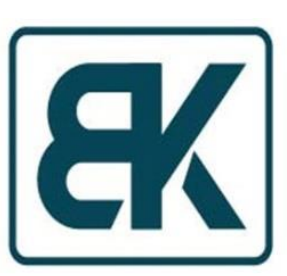

\section{Protein Engineering}

By Shyamasri Biswas*, PhD

Co Editor-in-Chief

Biotechnology Kiosk, 2, 5 (2020)

DOI: https://doi.org/10.37756/bk.20.2.5.2

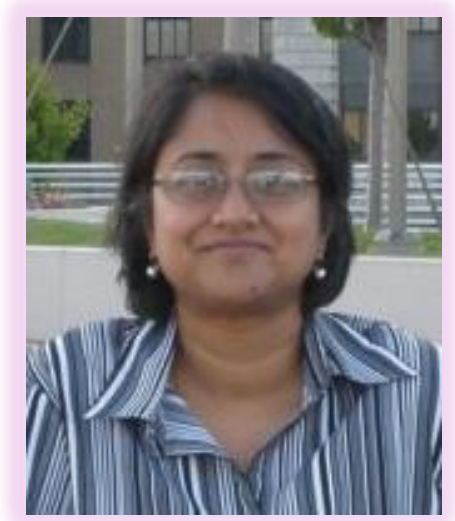

\section{Designer Proteins for New Vaccines and Therapeutics}

\begin{abstract}
Custom-designed or tailoring of proteins for specific applications is a highly active and growing area of research. A major part of this research is focused on protein engineering especially protein interfaces and interactions by employing biomolecular engineering tools. This research focus is partly due to the significance of protein interactions that are known to communicate critical information from the environment into cells to mobilize functional responses relevant to health and disease. Protein engineering can lead to custom-designed protein structures and biomaterials with the desired affinity, specificity, mechanism, or other properties that are of practical biomedical interests. Here, we describe a brief overview of protein engineering and some of the recent breakthroughs in biomedical applications of designer proteins. We discuss recent applications in new vaccine designs and advanced therapeutics for battling the challenges of complex diseases.
\end{abstract}

\section{*E-Mail: shyabiswas@biotechkiosk.com}




\section{Introduction}

The need for specifically engineered and rationally designed proteins with different functionalities has started the fascinating field of protein engineering. This field has provided many breakthroughs so far with wide-scale application potentials in industrial, biotechnological, and pharmaceutical sectors. Ongoing studies have shown tremendous promise of protein based therapeutics, new vaccines, and novel protein scaffolds with greater safety, improved efficacy, reduced immunogenicity and superior drug delivery with innovative biomedical formulations [1, 2]. To this end, researchers have proposed strategies to engineer protein interactions to modulate function using molecular engineering and also tailoring protein networks that exhibit robust plasticity that enables reprogramming of receptor interactions [3].

It is believed that the design strategies of innovative protein interface and engineered bio-orthogonal protein networks will lead to the development of advanced biologics and next generation research tools and therapies. The ongoing research has therefore, focused on developing new tools for protein engineering to realize the vast potential of protein materials. One such technique is directed evolution that has emerged as a powerful tool to improve biological systems. Directed evolution works based on mutation and selection. In this process, the encoding of DNA is mutated, and subsequently the resulting variants are screened and selected for specific functionality. This approach presents a promising avenue to produce novel protein materials [4]. In addition to directed evolution, a combination of various protein engineering approaches has been considered to create protein variants with a wide range of desired properties [2].

Further, computational methods have been shown to be of huge promise in protein engineering that can be leveraged for mechanism-driven interface design (Figure 1) [3]. To this end, researchers have integrated computational algorithms into the protein design process that has facilitated exciting new directions in immunotherapeutic development. For example, computational protein design can be employed to predict protein structures from amino acid sequences. This also includes engineering new proteins that enact desired functions. More importantly, it has been shown that computational methods can enable engineering of protein mechanisms, especially through design of neutralizing agents or allosteric modulators [3].

Here, we have described some of the important breakthroughs in protein engineering for new designs of vaccines and development of smart therapeutics.

\section{Artificial Proteins to Design New Vaccines}

Vaccines are known to trigger the immune system to produce antibodies. This protects humans against infection, and therefore, vaccines are considered the most effective interventions that prevent the spreading of infectious diseases. There has been an intense research focus on engineered protein designs particularly to obtain targeted neutralizing antibody $(\mathrm{nAb})$ responses for the development of new vaccines. De novo 
proteins are those proteins that are designed from the ground up. They are considered promising candidates in such vaccine designs [5].

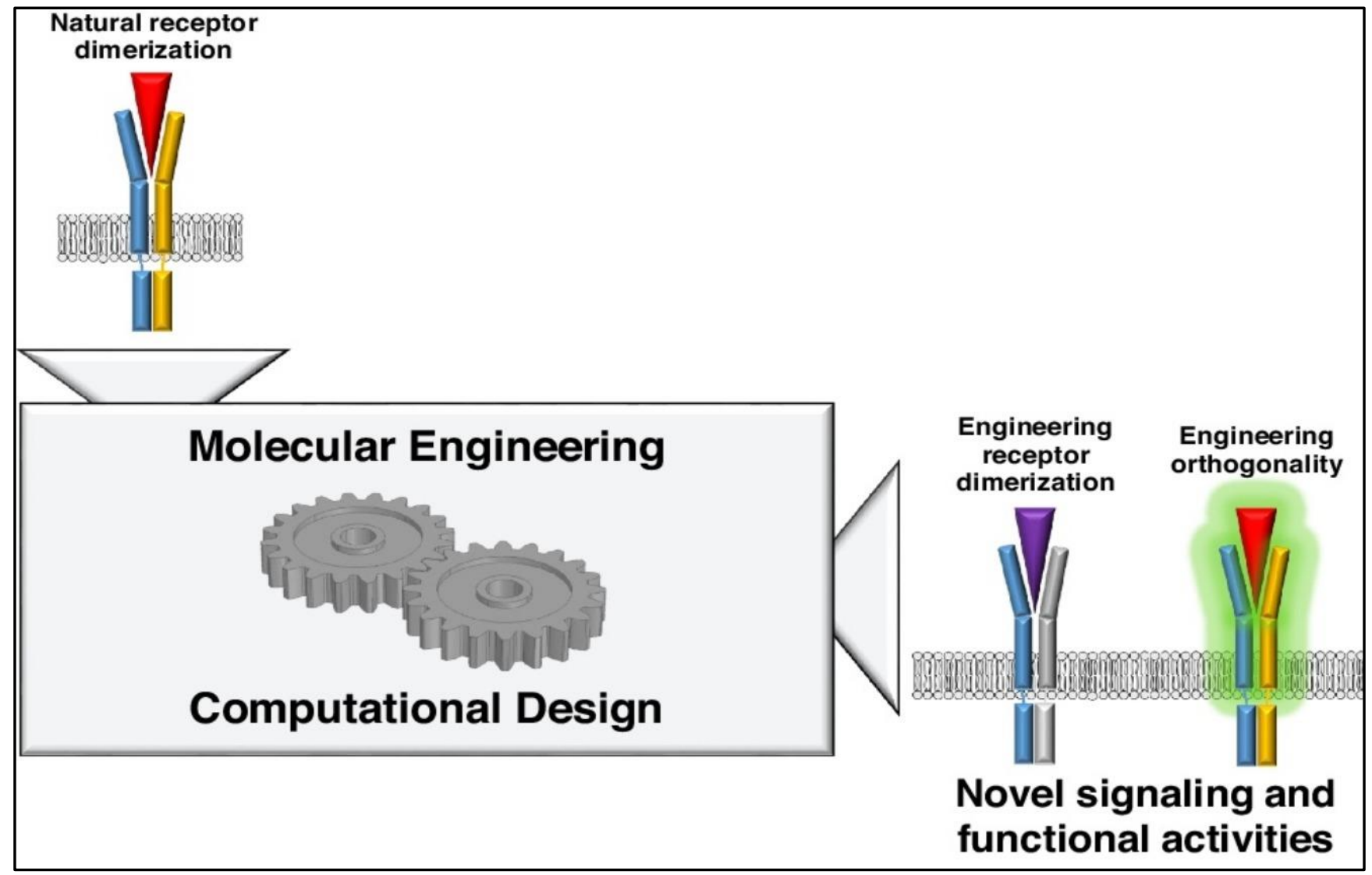

Figure 1: Schematic depiction of protein engineering using molecular engineering and computational design based approaches [Source: Current Opinion in Biotechnology (2019)].

De novo proteins that mimic a viral epitope outside the context of the native protein are of special interests due to their potential applications as immunogens that can induce targeted virus neutralizing antibodies (nAbs) in-vivo. However, de novo proteins exhibit regular and continuous structural patterns that are limited to mimicking the simplest epitopes. Thus, it's a challenge for the use of de novo proteins in epitope-focused immunogens that greatly limit their potential in the field of vaccine design [5].

Further, studies have shown that several major human pathogens only display a limited number of broadly neutralizing epitopes unlike respiratory syncytial virus (RSV) that are surrounded by strain-specific, non-neutralizing, or disease-enhancing epitopes [5]. This has presented a major research goal in engineering de novo proteins for vaccine development that seeks to trigger antibody responses with precisely defined epitope specificities. In addition, other specific requirements include constrained molecular features such as antibody lineage, complementaritydetermining region length, or binding angle [5]. 
Researchers addressed these challenges and limitations by employing de novo design approaches to engineer epitope-focused immunogens that mimicked irregular and discontinuous RSV neutralization epitopes.
They developed a strategy to design artificial proteins that very precisely instruct the body's immune system which antibodies to produce [5].

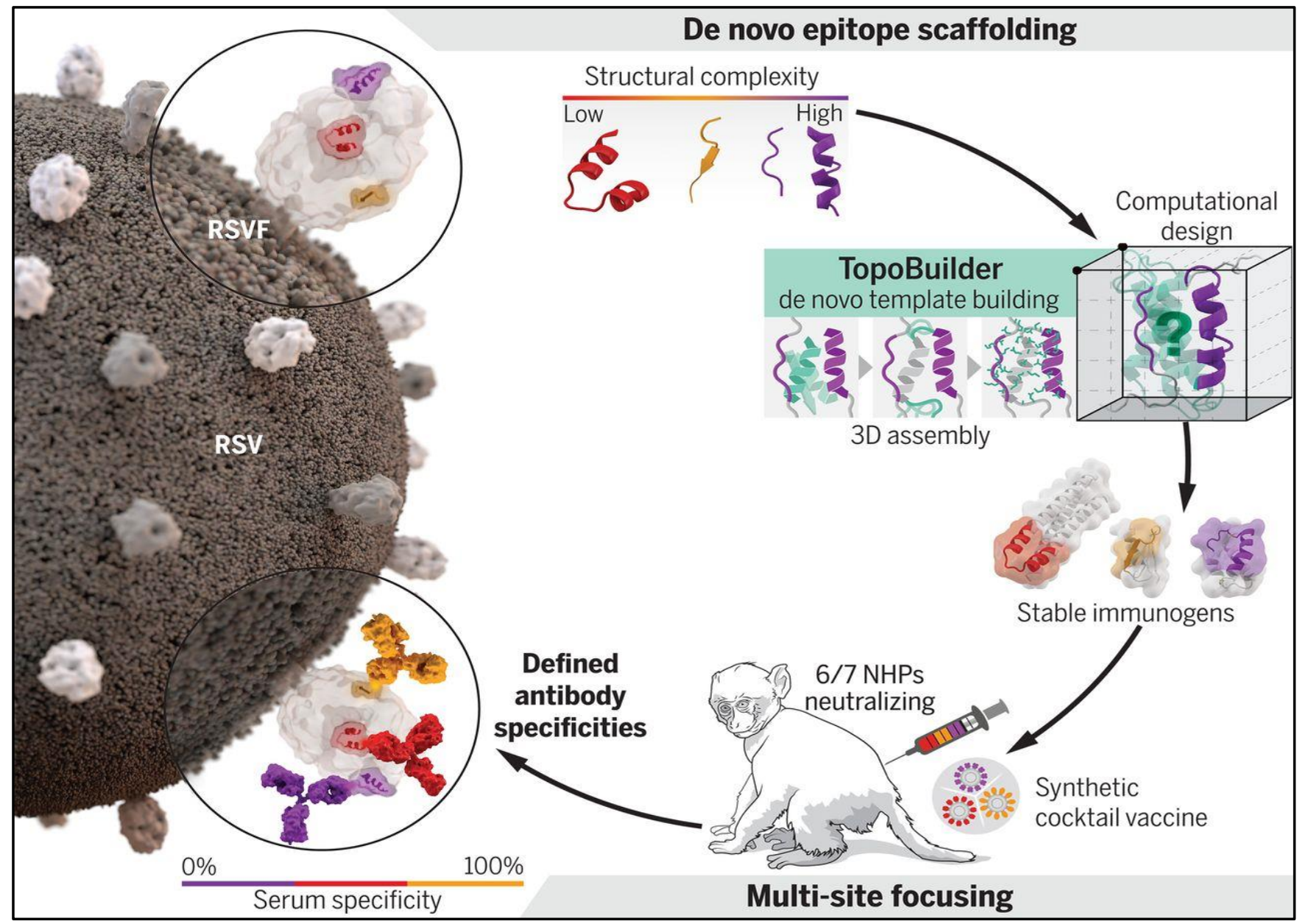

Figure 2: Schematic depiction of the de novo design of a trivalent cocktail vaccine that shows structurally complex RSV neutralization epitopes that are stabilized in de novo-designed proteins. The computational tool TopoBuilder is shown that builds customized protein topologies to stabilize functional structural motifs that are followed by folding and sequence design. This is subsequently applied to an in-vivo, a three-scaffold cocktail that induces focused RSV nAbs against the target epitopes [Source: Science (2020)].

In this strategy, researchers assembled protein topologies that were tailored to the functional motif. The aim was to enable the design of de novo proteins endowed with complex structural motifs. This approach was employed to develop an immunogen cocktail 
presenting three major antigenic sites of the respiratory syncytial virus (RSV) fusion protein (RSVF) in order to induce nAbs acting through precisely defined epitopes [5]. It was developed based on a novel computational design strategy, TopoBuilder, to build de novo proteins that presented complex structural motifs. TopoBuilder enabled to define and build protein topologies to stabilize functional motifs that were followed by in silico folding and sequence design using Rosetta (Figure 2) [5].

This study showed a new route to functionalizing de novo proteins that enables the assembly of customized protein topologies tailored to structural and functional requirements of the motif. This can be employed for epitope-centric vaccine design. Such a design strategy can be leveraged to gain control over induced antibody specificities in both naïve and primed antibody repertoires [5].

\section{Protein Therapeutics for Smart, Stimulus- Responsive Drug Systems}

In addition to new vaccine designs, one promising area of research in protein engineering is the development of smart, stimulus-responsive drug systems with improved clinical outcomes. This research has initiated a new field called protein therapeutics, which is expected to transform the metabolic drug landscape $[6,7]$. Although, engineering of protein therapeutics is a relatively new field, important discoveries in protein engineering tools are enabling to gain improved control over both pharmacokinetics and pharmacodynamics that are essential to take protein therapeutics to the next level. For example, innovative drugs based on stimulus-responsive protein therapeutics have been designed to be metabolized under targeted conditions. Further, researchers are using protein engineering to develop tailored smart therapeutics with biochemical logic [6-8].

Researchers have studied antibodydrug conjugates (ADC) that are considered the simplest form of stimulus-responsive protein therapeutics. In this therapeutics, a monoclonal antibody is used to target the conjugated drug to specific locations, such as cancer cells. In this process, a sensitive linker connecting the antibody and drug allows selective release of the drug [6].

The emergence of smart response drug systems has provided an exciting frontier of drug development that leverages advanced protein engineering capabilities. Researchers have envisioned smart response drug systems that can utilize feedback mechanisms to intelligently modulate a therapeutic effect in response to biomarkers or other relevant stimuli [6]. It is known that a drug is released slowly at an approximately constant rate in many existing delayed drug release systems. On the other hand, smart drug delivery systems differ from the delayed drug release process. Smart systems require the presence of external stimuli to turn on the release of a drug in activation-modulated release systems. Such systems may also require to modulate the extent of a drug that is released in feedbackmodulated release systems (Figure 3) [6]. 
a) Canonical drug delivery system

Effector
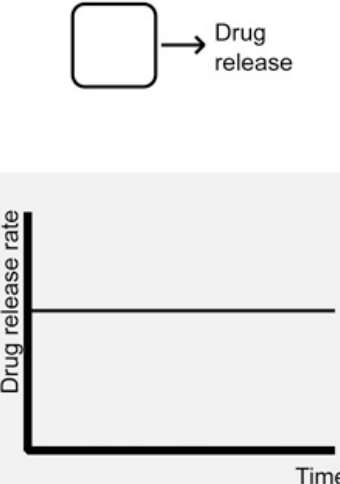

b) Smart drug delivery system

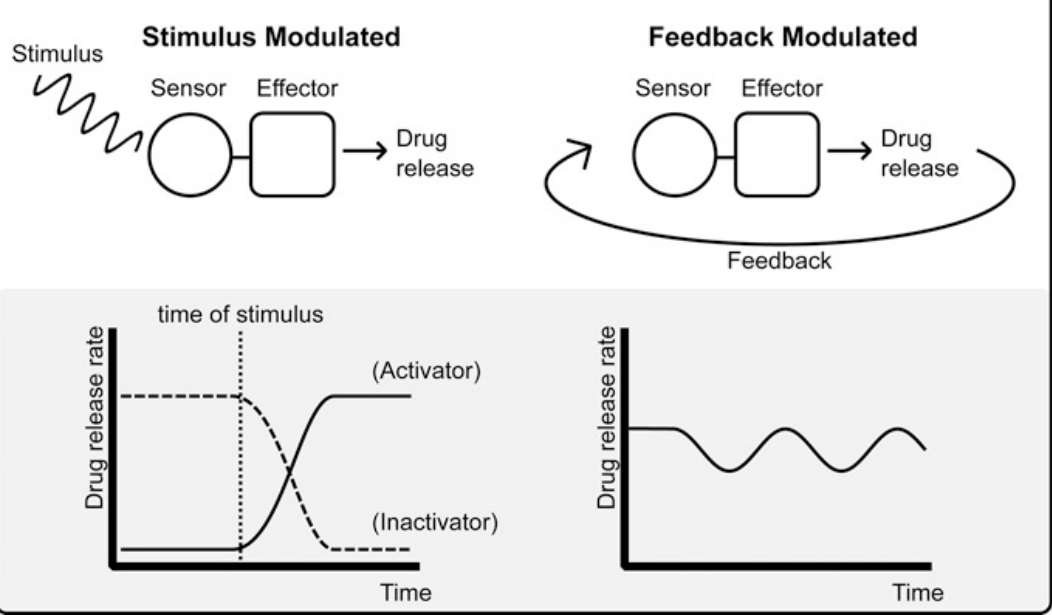

Figure 3: Schematics showing strategies for different drug delivery systems. (a) Existing drug delivery methods that typically involve releasing a drug at a relatively constant rate. (b) In contrast, smart drug delivery systems include both sensing and effector components, which allow drug release to either be activated or regulated in a feedback-dependent manner [Source: Curr Drug Metab. (2014)].

\section{Conclusion and Outlook}

The fascinating research field of protein engineering involving medicine and in particular pharmacology has shown tremendous potential for an exciting transition from small molecule chemical therapy to biological therapy. This exciting developments span from protein therapeutics, gene therapy to cellular therapy, where protein engineering is a central and enabling technology for all these biological therapies to combat complex disease. Ongoing studies have suggested that for protein therapeutics, a robust control of half-life and immunogenicity needs to be achieved to accomplish clinical goals. It is anticipated that protein engineering will continue to impact these areas for next generation vaccine and therapeutics developments.

It is also anticipated that future therapeutics will be much broader by taking advantage of the full potential of protein engineering. In this regard, one area in engineering protein therapeutics with huge growth potential is expected to be stimulusresponsive for targeted activation and/or targeted neutralization, which will revolve around the modulation of protein function and stimulus-responsive function. This will further be impacted by the ongoing advances in gene therapy and other intracellular protein delivery methods that can improve various protein design constraints. Future research is expected to focus on better understanding of protein molecular recognition, allostery, and catalysis that will pave the way for further 
development of computational protein design methods.

\section{References}

1. Malgosia M. Pakulska, Shane Miersch, Molly S. Shoichet, Designer protein delivery: From natural to engineered affinity-controlled release systems, Science, 351, Issue 6279, aac4750 (2016), DOI:

https://doi.org/10.1126/science.aac4750

2. Brindha J., Balamurali M. M. and Kaushik Chanda, Evolutionary approaches in protein engineering towards biomaterial construction, RSC Adv., 9, 34720-34734 (2019), DOI: https://doi.org/10.1039/C9RA06807D.

3. Patrick J Krohl, Seth D Ludwig and Jamie B Spangler, Emerging technologies in protein interface engineering for biomedical applications, Current Opinion in Biotechnology 60:82-88 (2019), DOI: https://doi.org/10.1016/j.copbio.2019.01.0 $\underline{17}$.

4. Anton Kan and Neel S. Joshi, Towards the directed evolution of protein materials, MRS Commun., 9(2): 441-455 (2019), DOI: $\underline{10.1557 / \mathrm{mrc} .2019 .28}$.
5. Fabian Sesterhenn, Che Yang, Jaume Bonet, Johannes T. Cramer, Xiaolin Wen, and Bruno E. Correia, De novo protein design enables the precise induction of RSV-neutralizing antibodies Science, 368, Issue 6492, eaay5051 (2020), DOI: $\underline{10.1126 / \text { science.aay5051. }}$

6. Peter H. Tobin, David H. Richards, Randolph A. Callender, and Corey J. Wilson, Protein Engineering: A New Frontier for Biological Therapeutics, Curr Drug Metab., 15(7): 743-756 (2014), DOI: $\underline{10.2174 / 1389200216666141208151}$ $\underline{524}$

7. Steven M. Jay and Richard T. Lee, Protein Engineering for Cardiovascular Therapeutics, Circulation Research, 113, Issue 7, 13, 933-943 (2013), DOI: https://doi.org/10.1161/CIRCRESAHA.11 $\underline{3.300215}$.

8. Michaela Gebauer and Arne Skerra, Engineered Protein Scaffolds as NextGeneration Therapeutics, Annual Review of Pharmacology and Toxicology, 60:391415 (2020), DOI: https://doi.org/10.1146/annurevpharmtox-010818-021118 


\title{
REVIEW ARTICLE
}

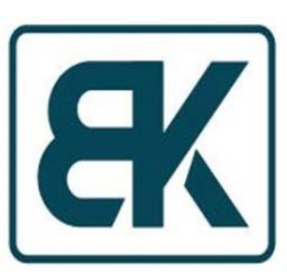

Aging

By Shripriya Singh, PhD

Contributing Editor

Biotechnology Kiosk, 2, 5 (2020)

DOI: https://doi.org/10.37756/bk.20.2.5.3

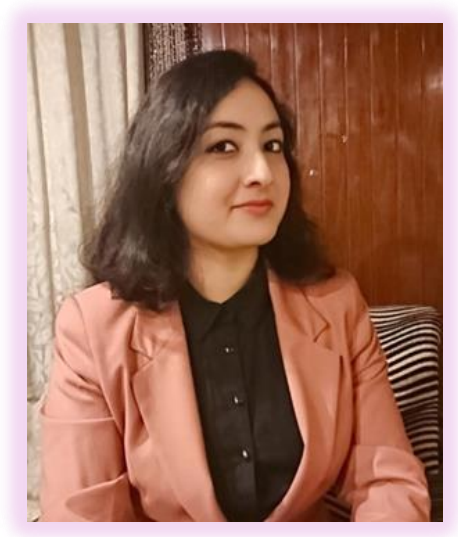

\section{Worries make you look older: unraveling the science that connects stress with graying in human beings}

\begin{abstract}
Once considered a taboo, mental health is a trending health topic now days and is considered one of the crucial aspects of good health. Neuroscience and medical advances can be credited for busting the longstanding myths and dogmas associated with brain health. Stress is one of the most common factors that affect the mental well being and has been long associated with graying in human beings, however without any significant medical or scientific proof. Here, we describe this concept of premature aging that has intrigued researchers for a long time. A recent study provided evidence in favor of the stress based theory of aging. Mouse was used as a model organism to study how acute stress leads to hair graying. A combination of denervation, chemogenetics, cell ablation, adrenalectomy and knockout of the adrenergic receptor specifically in melanocyte stem cells was used to check potential targets. After eliminating different possibilities it was confirmed that acute stress leads to the activation of the sympathetic nervous system which in turn causes permanent damage to tissue regeneration via nonreversible depletion of melanocyte stem cells (MeSCs) residing in the bulge and hair germ region of the follicle which are mainly responsible for the regeneration of pigmented hair.
\end{abstract}

Keywords: Stress; graying; aging; melanocyte stem cells (MeSCs); sympathetic nervous system.

\section{*E-Mail: shripriyasingh@gmail.com}

To cite this article: Singh S; Worries make you look older: unraveling the science that connects stress with graying in human beings, Biotechnology Kiosk, Vol 2, Issue 5, PP: 18-23 (2020); DOI:

https://doi.org/10.37756/bk.20.2.5.3 


\section{Introduction}

According to The World Health Organization (WHO) human health is defined as "a state of complete physical, mental and social wellbeing and not merely the absence of disease or infirmity". A lay man often confuses good health with only a fit disease free body although in reality good health also refers to a healthy mind. Mental health has become a trending topic and has been garnering attention from all over the world. Earlier people shied away from discussing their mental health issues and rightly so because it was one of the most frequently misunderstood aspects of human health. Neuroscience and medical advances can be credited for busting the longstanding myths and dogmas associated with brain health. Today we are equipped to understand neuronal diseases much better and the complexity of the human brain has been largely decoded. We have developed an understanding of neuronal development, neurodegeneration and neuroregeneration. There are various neurodegenerative diseases and brain associated disorders which have been the focus of scientific and medical research. Several adverse conditions of the brain have been medically acknowledged and find widespread attention from not only a disease point of view but also as an important aspect of human personality.

Eminent personalities and celebrities have also positively highlighted the importance of mental health via anecdotes and social media posts. Thanks to the awareness raised, people are now realizing that visiting a psychologist and psychiatrist does not necessarily mean a person is mentally ill. However when we talk about the mental health issues one of the first words that draw our attention is stress. Stress is a word we frequently use in our everyday life, sometimes without even realizing the actual medical meaning of the term.

Biologically speaking stress is an emotional, mental or a physical factor that causes bodily or mental tension. Various external environmental, social and psychological factors can lead to stress. Similarly internal factors such as disease or surgeries are also responsible for stress. Hectic work schedules and unhealthy lifestyles are the major reasons which cause stress. We have often heard people complaining that stress causes graying of hair and there are several anecdotes cited as examples. However so far, no medical research had ever confirmed the theory with conviction that whether stress causes graying or not.

For the very first time a group of Harvard researchers have given scientific evidence in favor of the theory and discovered the underlying mechanism. In the current article we shall briefly discuss the salient findings of their research work which was recently published in the journal Nature [1].

\section{Stress and graying: the neuroscience behind the theory}

It is well known that stress has an overall impact on the human body comprising various tissues, however graying hair is one of the most obvious and visible changes that are associated with the same. The hair follicle comprises three stages i.e. growth, degeneration and rest which are also known as anagen, catagen and telogen phases respectively. Hair follicle stem cells (HFSCs) 
and melanocyte stem cells (MeSCs) residing in the bulge and hair germ region of the follicle are mainly responsible for the regeneration of pigmented hair. While HFSCs are responsible for the production of new hair, MeSCs are largely responsible for providing the pigment to the newly generated hair follicles via melanocytes [2]. Thus melanocyte lineage can be used as a suitable model to investigate how stress and tissue regeneration are interlinked.

Since stress affects the entire body it was challenging for the researchers to narrow down and pinpoint which specific systems were involved. Studies were carried out in mice with black coat color and three different approaches were used to model stress: chronic unpredictable stress, restraint stress and nociception-induced stress (which was achieved through an injection of resiniferatoxin (RTX)) [3]. All three procedures led to the formation of white (unpigmented) hair over time but nociceptioninduced stress produced the most rapid and pronounced effect-many new hair that formed in the next hair cycle after RTX injection became unpigmented. In order to select the target system several theories were proposed and then cross checked for results. The research team previously hypothesized that stress induced immune attack leads to hair graying. To check the involvement of the immune system, RTX was injected into Rag1 mutant mice which lack both $B$ and T cells and into CD11b-DTR mice, in which myeloid lineages had been ablated by diphtheria toxin. RTX injection into these immune-deficient mice still resulted in formation of white hair, thus ruling out the possible involvement of the immune system [1].

All forms of stress are known to increase the levels of corticosterone and noradrenaline in the blood and therefore this hormone became the next target for research. Thus using a combination of denervation, chemogenetics, cell ablation, adrenalectomy and knockout of the adrenergic receptor specifically in melanocyte stem cells it was confirmed that the stress induced loss of melanocyte stem cells is independent of adrenal stress hormones or immune attack [1].

Once the different possibilities were eliminated, researchers narrowed down on the sympathetic nervous system, which is responsible for the body's fight-or-flight response [4]. The sympathetic nerves innervate the melanocyte stem-cell niche and branch out into each hair follicle on the skin. Stress induces the activation of these nerves which in turn release a burst of the neurotransmitter noradrenaline (also known as norepinephrine). This neurotransmitter is rapidly taken up by the nearby pigmentregenerating stem cells. This causes the quiescent melanocyte stem cells to proliferate rapidly, and is followed by their differentiation, migration and permanent depletion from the niche. If the proliferation of melanocyte stem cells can be transiently suppressed, the stress induced hair graying can be prevented. Figure 1 shows the three dimensional reconstructed image depicting the sympathetic innervations around pigment-regenerating stem cells. 


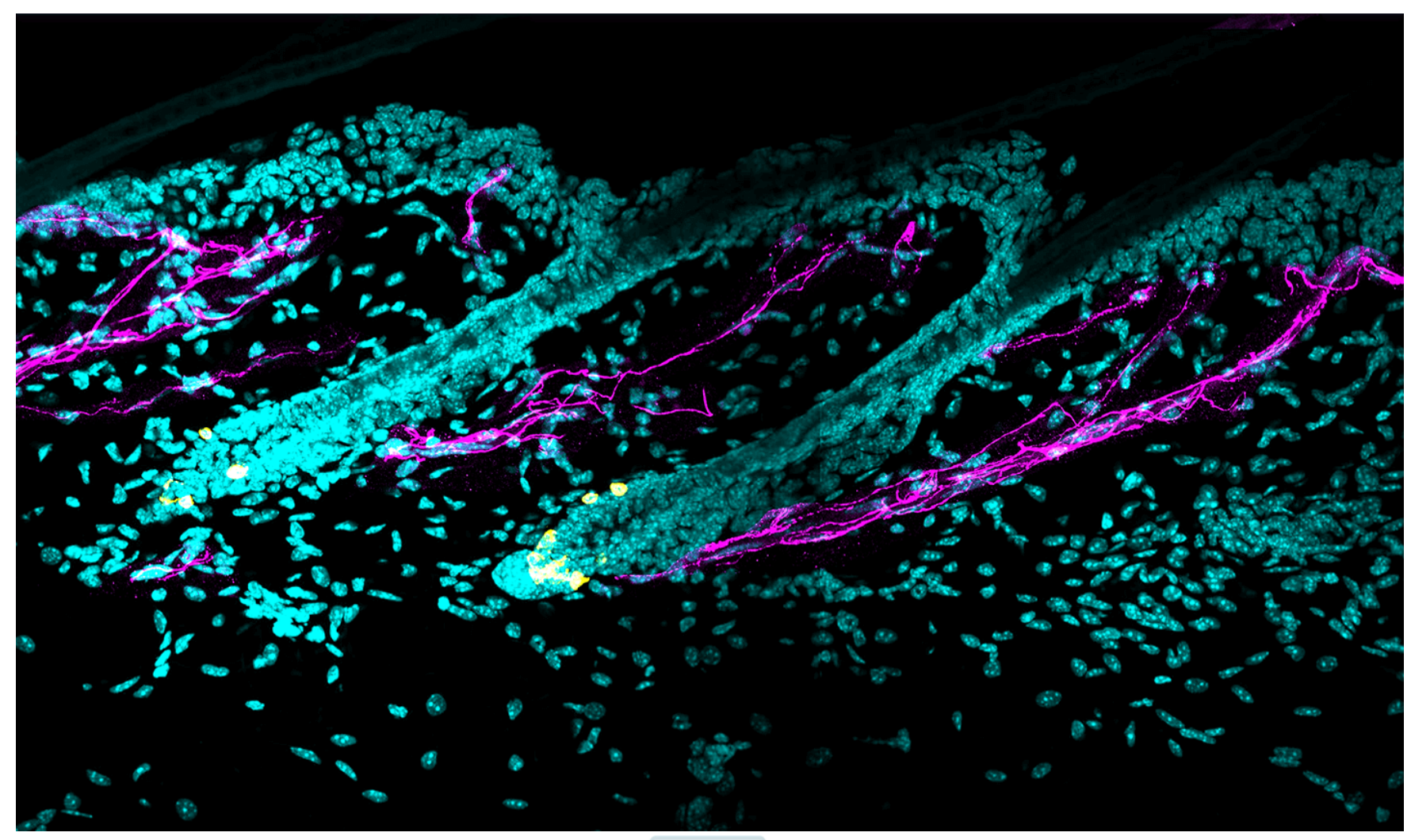

Figure 1: $3 D$ reconstructed image showing the elaborate sympathetic innervations (depicted in magenta) around pigment-regenerating stem cells (depicted in yellow). The hyperactivation of the sympathetic nervous system is triggered by acute stress which results in a burst release of neurotransmitter norepinephrine. The neurotransmitter then rapidly depletes the pigment-regenerating stem cells leading to hair graying. [Source: Hsu Laboratory, Harvard University 2020, (https://hscrb.harvard.edu/news/solving-a-biological-puzzle-how-stresscauses-gray-hair/)].

The study results clearly show that acute stress triggers neuronal activity that can drive a prompt and permanent loss of somatic stem cells. Once the reservoir of naïve MeSCs is exhausted there is no means of replenishing the pigment generating cells and thus gradually graying becomes an irreversible process. Acute stress triggers beneficial and transient fight-or-flight responses which are critical for the survival of living beings but any factor that can disturb the homeostasis of the body will have negative side effects too. The current study is an example that illustrates that overall physiological state of the organism directly influences the maintenance of somatic stem cells. The results clearly highlight that stress leads to the activation of the sympathetic nervous system which in turn causes permanent damage to tissue regeneration via non-reversible depletion of somatic stem cells. Figure 2 summarizes the concept of how stress triggers graying in mice. 

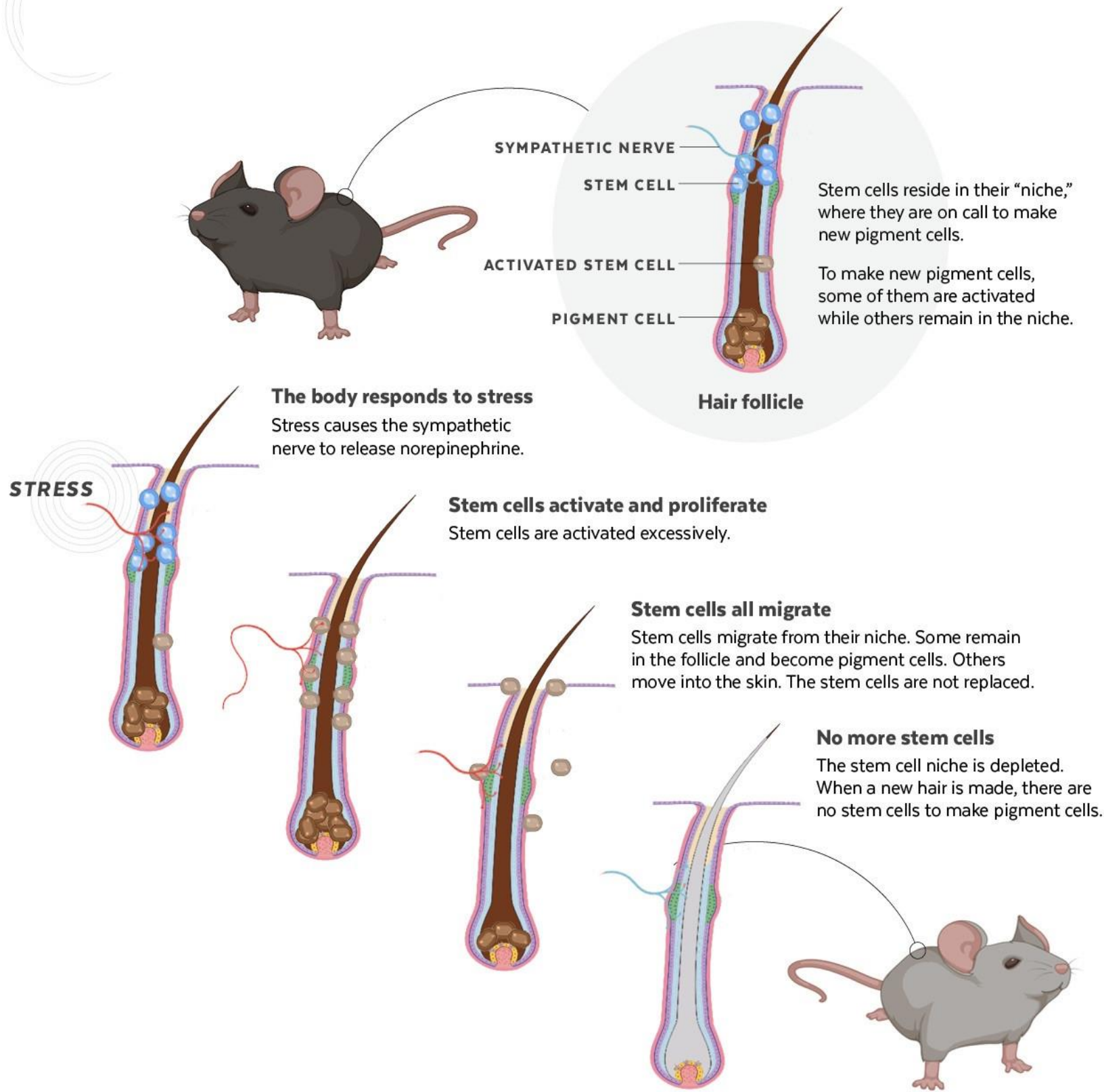

Figure 2: schematic of how stress makes hair turn gray in mice. [Source: Judy Blomquist/ Harvard University (https://news. harvard.edu/gazette/story/2020/01/new-findings-suggest-howstress-may-trigger-gray-hair/)].

\section{Conclusion and outlook}

The current study has clearly established the link between stress and graying and has opened new avenues for further research. As essentially all the organs are innervated by the sympathetic nerves acute stress might 
possibly affect many different tissues through neuronal signals. The connection between pigment-producing cells and the nervous system has been evolutionarily conserved and several interesting examples are found in nature. For example Cephalopods like octopus, cuttlefish and squid have unique coloration systems that allow them to change color for communication or camouflage. Similarly chromatophores (pigment producing cells) are controlled by neuronal activities which allows rapid changes in color in response to predators or threats [5]. Graying is a natural aging process but it is often confused with old age. It is very natural for teenagers and young adults to become conscious about their looks when they notice grey hair. It leads to low self-esteem and anxiety which again triggers stress. Thus it is a never ending cycle. Although stress is not the only reason for graying, genetics, disease and other unknown factors are also responsible. However, stress is the simplest and most obvious reason that can be taken into consideration. We cannot completely eliminate stress causing situations around us but can definitely make efforts to improve our mental health and well-being. To conclude we would suggest keep worries at bay to avoid growing grey.

\section{References}

1. Zhang B, et al. (2020) Hyperactivation of sympathetic nerves drives depletion of melanocyte stem cells. Nature 577(7792):676-681. DOI: 10.1038/s41586$\underline{020-1935-3}$
2. Rabbani P, et al. (2011) Coordinated activation of Wnt in epithelial and melanocyte stem cells initiates pigmented hair regeneration. Cell 145(6):941-955. DOI: $\underline{10.1016 / j . c e l l .2011 .05 .004}$

3. Baral P, et al. (2018) Nociceptor sensory neurons suppress neutrophil and $\gamma \delta \mathrm{T}$ cell responses in bacterial lung infections and lethal pneumonia. Nature medicine 24(4):417. DOI: $\underline{10.1038 / \mathrm{nm} .4501}$

4. Ulrich-Lai YM \& Herman JP (2009) Neural regulation of endocrine and autonomic stress responses. Nature reviews neuroscience

DOI: $10.1038 / \mathrm{nrn} 2647$

5. Reed CM (1995) The ultrastructure and innervation of muscles controlling chromatophore expansion in the squid, Loligo vulgaris. Cell and tissue research 282(3):503-512.

DOI: $10.1007 / \mathrm{BF} 00318882$ 


\title{
REVIEW ARTICLE
}

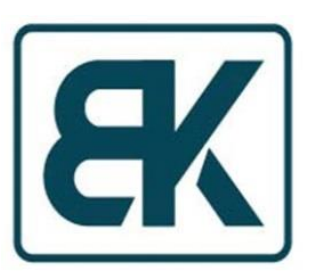

\section{Myocardial complications and COVID-19 infections}

\section{Sangeeta Chakraborty, PhD}

National Jewish Health, Denver, Colorado, USA

Biotechnology Kiosk, 2, 5 (2020)

DOI: https://doi.org/10.37756/bk.20.2.5.4

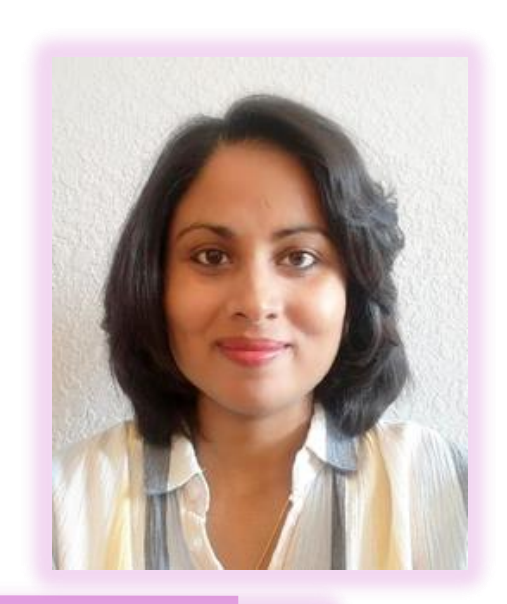

\section{Impact of COVID-19 on cardiovascular patients}

\begin{abstract}
The novel coronavirus disease (COVID-19) pandemic, caused by SARS-CoV-2, has affected people's health in more than one way with catastrophic impacts on primary healthcare systems across the globe. However, the rapid expansion of knowledge over the last few months, supported by rigorous scientific research, has enabled our understanding of the mechanism(s) of COVID-19 and potential therapeutics. Initial infection of the upper respiratory tract by SARSCoV-2 is mediated by binding of the virus to host expressed angiotensin-converting enzyme 2 receptor (ACE2). While viral pneumonia is one of the predominant manifestations of COVID-19, current evidence shows that the virus also affects multiple organs, especially the cardiovascular system. Comorbidities such as hypertension, diabetes, and coronary heart disease, not only result in worsening outcomes but also account for high death counts among infected patients. Serious cardiovascular complications such as cardiac injury, heart failure, and arrhythmias are often seen in the severe hospitalized cases of COVID-19; such cases predominantly feature hyper inflammation, cytokine storm along with elevated cardiac injury biomarkers. Whether viral invasion through ACE2-expressed in heart cells-is responsible for the development of new cardiovascular dysfunction in COVID-19 patients without any history of heart disease, remains under investigation. Other concerns have also surfaced regarding the use of renin-angiotensin system (RAS) inhibitors like angiotensin receptor blockers (ARBs) and ACE inhibitors (ACEIs), potentially increasing the severity of COVID-19. Here, we review current research and epidemiological data on SAR-CoV-2 infection and its clinical manifestations with a focus on cardiovascular complications and mechanisms of injury. Current guidelines over the use of RAS inhibitors in the context of the speculated role of ACE2 in COVID-19 patients are also discussed.
\end{abstract}

\section{*E-Mail: sangeeta1986@gmail.com}

To cite this article: Chakraborty S; Impact of COVID-19 on cardiovascular patients, Biotechnology Kiosk, Vol 2, Issue 5, PP: 24-33 (2020); DOl: https://doi.org/10.37756/bk.20.2.5.4 


\section{Introduction}

Since the identification of novel coronavirus infection in a small cluster of pneumonia patients in Wuhan, China, SARS-CoV-2 (Severe Acute Respiratory Syndrome COronaVirus 2)-the virus responsible for COronaVIrus Disease (COVID-19)-has been on a global rampage killing more than 3 million people in its wake, stretching healthcare systems to their limits and causing severe socioeconomic loses. Even with stringent containment measures such as social distancing, rigorous disinfection, and lockdown, the transmission rates have been difficult to reduce. The US, which has been hit hard by the pandemic, saw an unprecedented rise in the number of COVID19 cases between March and April, putting the nation on top for the highest number of cases [1].

Since the outbreak, we have gathered a substantial amount of information about SARS-CoV-2 genetics and pathology. Genome sequencing and phylogenic analysis revealed that SARS-CoV-2 is a positive-stranded RNA $\beta$-coronavirus. It has $80 \%$ nucleotide homology to its ancestor SARS-CoV that caused the 2002 SARS outbreak and $50 \%$ nucleotide homology to MERS-CoV (Middle East Respiratory Syndrome Coronavirus) that was responsible for the 2012 outbreak [2, 3]. Consequently, SARS-CoV-2 employs the same receptor to enter cells as SARS-CoV: the transmembrane Angiotensin-Converting Enzyme II (ACE2) receptor [3]. Once inside the upper respiratory tract, SARS-CoV-2 binds to ACE2 in the nasal epithelium through its surface spike protein (S), and through a serine protease cleavage event, is endocytosed into the host cell [4]. Tissue ACE2 expression is broad and occurs in a variety of cell types. In the respiratory system, it is predominantly expressed in the alveolar type II (AT2) cells of the lungs and to a variable extent in other cells like heart, kidneys, intestinal epithelium, and vascular endothelium [4].

Clinical symptoms of SARS-CoV-2 are diverse and overlap with those associated with other viral respiratory infections; however, the rapid transmission rates combined with a higher risk of mortality distinguish COVID-19 cases from influenza. Symptomatic COVID-19 can range from mild symptoms like cough, fever, sore throat to severe manifestations like pneumonia, dyspnea, and acute pulmonary stress (ARDS) that often require admittance to hospital [5]. Most ICU cases of critically ill patients present with life-threatening conditions, such as acute cardiac injury, severe lymphopenia, shock, and abnormal inflammatory response that most often progress to multi-organ failure and death [5, $6,7]$. While a large proportion of COVID-19 infections are not severe, hospitalized cases commonly have a critical or fatal disease. In a report from the Chinese Center for Disease Control and Prevention that included 44,672 confirmed COVID-19 cases, the overall case fatality rate (CFR) - the number of deaths divided by the number of confirmed caseswas $2.3 \%$, but in the critically ill population, the CFR rose to a staggering $49 \%$ [8]. In the worst affected countries, the CFR is anywhere between $6-16 \%$ [9], and there is a strong correlation of mortality rate with advanced age [6]. In Italy, the CFR among patients aged 80 years or older rose to almost 
$20 \%$ owing to a greater proportion of geriatric population in the country $(23 \%$ of the population $>65$ ) [10]. With more diagnostic tests starting to become available and accessible in recent times, the case fatality rate, which depends on the number of infected cases, is more likely to reflect an accurate estimation of the level of death in the COVID-19 pandemic.

Epidemiological and meta-analyses data from the first two months of the outbreak in China shows a consistent association of pre-existing cardiovascular disease (CVD) and relevant risk factors with the severity of COVID-19. A study on a pool of 1527 COVID19 patients from six different hospitals in China reported both hypertension and diabetes to be twice as prevalent in ICU admitted COVID-19 cases than in their nonICU counterparts [11]. In yet another early meta-analysis of seven studies, including 1576 COVID-19 cases, the most frequent comorbidities were hypertension $(21.1 \%)$ and diabetes $(9.7 \%)$, followed by cardiovascular disease $(8.4 \%)$ and respiratory disease $(1.5 \%)$ [16]. It is prudent to conclude, given the evidence, that pre-existing morbid ailments in COVID-19 patients contribute to an elevated risk of death [2, 14].

\section{Do cardiovascular comorbidities increase mortality among COVID-19 patients?}

The most common underlying health issues found to result in poorer prognosis and mortality among COVID-19 patients are hypertension, diabetes mellitus (DM), and coronary heart disease $[4,12,14]$. Comorbid patients often require intensive care treatments compared with those who have no underlying medical issues [12]. A large case- series report from the Chinese center for disease control and prevention, including 44,672 confirmed COVID-19 cases showed an increase in the mean death rate $(2.3 \%)$ with comorbid conditions: $10.5 \%$ for cardiovascular disease, $7.3 \%$ for diabetes, $6.3 \%$ for chronic respiratory disease, $6.0 \%$ for hypertension. Non-critical COVID-19 patients with no underlying comorbidities had no death [13]. As per reports from the National Health Commission of China, on COVID-19 patients who died, $35 \%$ had a history of hypertension, and $17 \%$ had a history of coronary heart disease [15]. A multi-center meta-analysis including a cohort of 1099 COVID-19 patients from 552 hospitals in China revealed that among the severely sick, $38.7 \%$ had comorbid health conditions, specifically, $23.7 \%$ with hypertension and $16.2 \%$ with diabetes. A high death rate of $8.1 \%$ in the severe cases vs. only $0.1 \%$ in the non-severe cases revealed that underlying cardiovascular conditions account for a large proportion of fatalities from COVID-19 [17].

Several mechanisms are thought to be responsible for the heightened susceptibility seen in severe COVID-19 infections with underlying CVD (Figure 1). Some of the possibilities could be: immune dysregulation due to an imbalance in type 1 and type $2 \mathrm{~T}$ helper cell response, insufficient tissue oxygenation during pulmonary distress, and adverse effects originating from treatment regimens that are yet to be evaluated in the setting of this pandemic [18]. As evidence suggests, SARS-Cov-2 seems to aggravate complications in patients with pre-existing cardiovascular disease, but does it induce new cardiac pathologies also? 
Could SARS-CoV-2 infection be responsible for promoting new heart injuries?

Some initial case studies originating from China reported high levels of high-sensitivity cardiac troponin I (hs-cTnl) and troponin T (TnT) in critically ill COVID-19 patients, suggesting complications involving acute myocardial injury [12, 19]. Hs-cTnl and TnT are biomarkers of acute cardiac injury and correlates well with other diagnostic markers of myocardial shock, such as high-sensitivity $\mathrm{C}$-reactive protein and $\mathrm{N}$-terminal pro-brain natriuretic peptide (NT-proBNP) [18]. In the aforementioned study reported by the National health commission of China, $11.8 \%$ of hospitalized COVID-19 patients, without underlying CVD, died of newly developed cardiac injury or cardiac arrest, as evidenced by their high levels of serum hs-cTnl [15]. A single-center case report including 187 patients with confirmed COVID-19 revealed that elevated TnT mediated myocardial injury claimed more lives than underlying CVD: a death rate of $37.5 \%$ was noted for those without CVD but with high TnT vs. 13.33\% for those with underlying CVD and normal TnT [20].

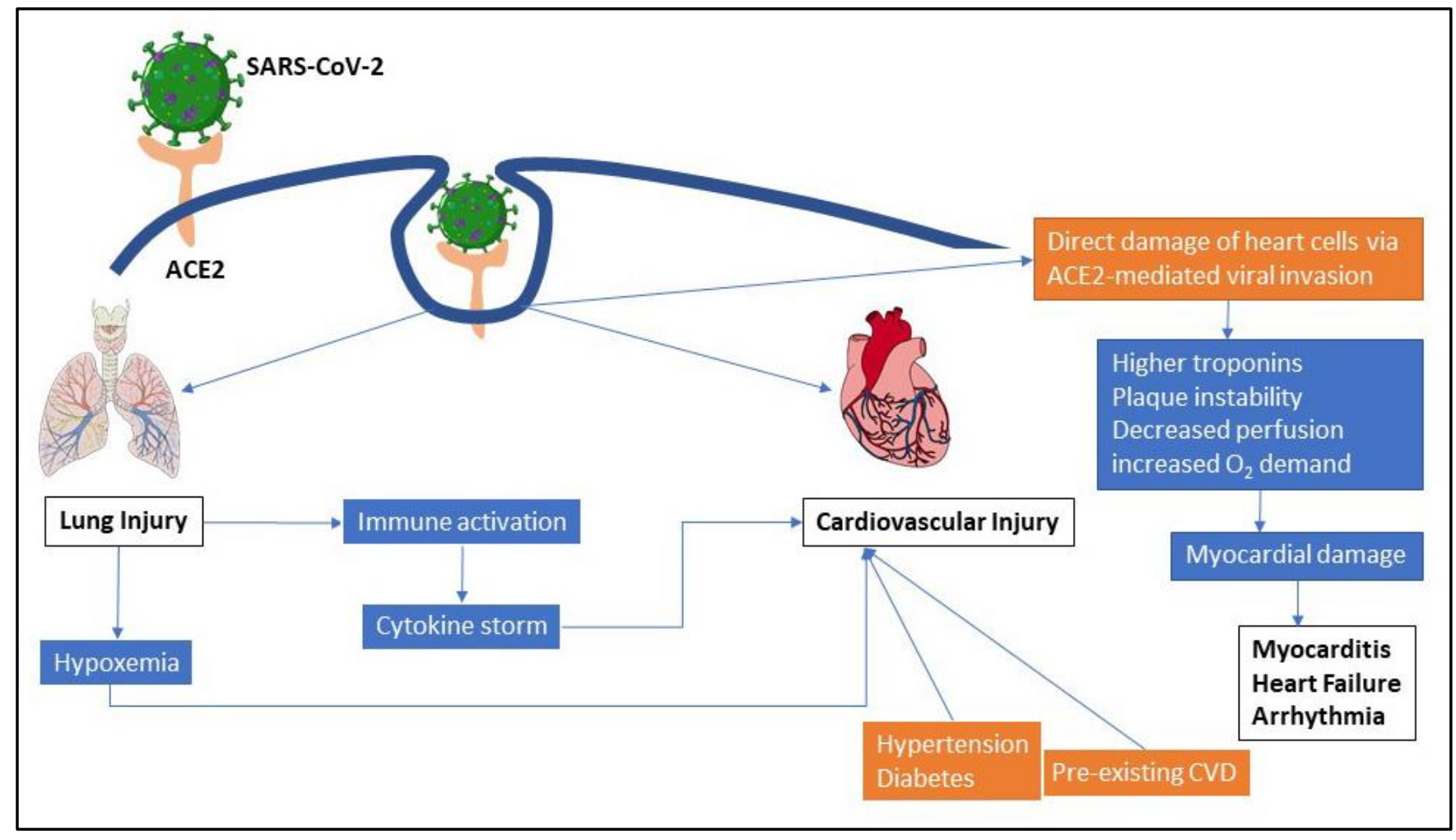

Figure 1: Potential pathways of COVID-19 associated pulmonary and cardiovascular injury. Infection of the respiratory tract by SARS-CoV-2 leads to systemic inflammation and dysregulated immune response which subsequently progresses into a "cytokine storm". Similarly vial invasion of cardiac myocytes may directly lead to myocardial dysfunction, arrhythmias and eventually heart failure. Pre-existing conditions like hypertension and diabetes can further complicate viral disease outcomes. 
Currently, there is not enough data on the pathophysiological mechanisms employed by SARS-CoV-2 in causing heart injury. Some speculation, however, correlates increased ACE2 expression on cardiomyocytes - that could lead to direct myocardial infection by SARS-CoV-2-with the myocardial injury that is often seen in severe COVID-19 [6, 18, 21].

\section{ACE2 and SARS-CoV-2-facts and speculations.}

Myocardial complications in COVID-19 infections, along with the fact that SARSCoV-2 uses ACE2 to enter host cells-which is highly expressed in the heart-have fueled debates among researchers on the possible impact of ACE2 on COVID-19 cases with new or existing CVD.

ACE2, a transmembrane peptidase is a component of the renin-angiotensin system (RAS); RAS is a complex endocrine cascade regulating blood pressure, fluid volume, and electrolyte balance. ACE2 is expressed in a variety of tissues, but at an organ level, the highest activity is seen in the upper Gl tract (ileum) and kidney followed by heart, brain stem, lung, vascular endothelium, stomach, and mucosal surfaces [4, 22]. ACE2 counters the deleterious activity of a hyperactive RAS that promotes hypertension, oxidative stress, and inflammation by converting ANG II (angiotensin II-a vasoconstricting octapeptide) to Ang-(1-7) [angiotensin-(17) - a vasodilating heptapeptide]. A higher level of ACE2 receptor showed improved cardiac function post myocardial infarction and better glycemic control in diabetes, possibly through the cardioprotective, antiinflammatory, and anti-fibrotic actions of Ang-
(1-7) [22, 23]. ACE2 knock out mice developed severe lung injury [24].

How ACE2 is modulated in the context of SARS-CoV-2 infection is not yet fully known. Studies on SARS-CoV revealed that viral infection reduced ACE2 in mice, leading to an increase in ANG II, which promoted pulmonary vascular permeability, inducing pulmonary edema, and lung dysfunction [6]. At present, preliminary research on SARSCoV-2 yields similar data: virus-induced downregulation of ACE2. Either viral internalization by endocytosis with ACE2 reduces surface ACE2 [22] or proteasemediated ACE2 shedding during the infection (identical to SARS-CoV infection) may bring down the level [23]. In either case, loss of ACE2 would disrupt the generation of cardioprotective Ang-(1-7), increasing the ratio of ANG II:Ang-(1-7) and, in theory, could potentiate pulmonary and cardiac damage in COVID-19 infections. Indeed, this could partially explain the cardiopulmonary manifestations, such as myocardial injury, respiratory dysfunction, and hyperinflammation seen in COVID-19 patients [23].

On the one hand, ACE2 is cardioprotective and prevents lung injury, and on the other, it serves as the portal of entry for SARS-CoV2 , then is ACE2 beneficial or detrimental in the current pandemic situation? Although definitive answers to that are lacking, researchers predict that a lower ACE2 in the nasal epithelium could help restrict SARSCoV-2 infiltration in the body, but at the same time, a higher ACE2 in the lower respiratory tract and heart might help reduce SARSCoV-2 -mediated risks of lung and myocardial injuries among vulnerable COVID-19 patients [22, 25]. 

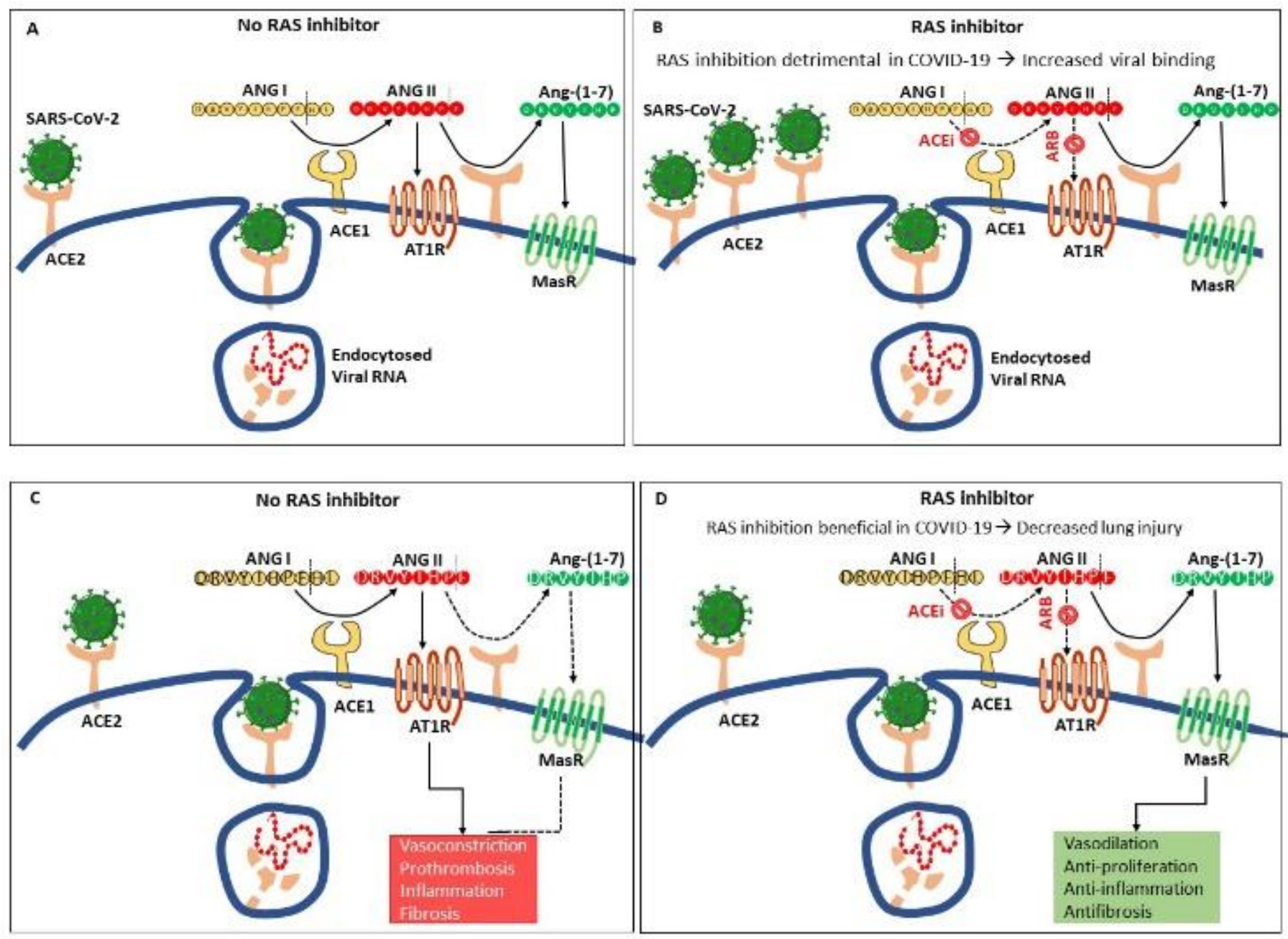

Figure 2: Possible mechanisms by which inhibition of the renin-angiotensin system (RAS) with an angiotensin-converting enzyme inhibitor (ACEi) or angiotensin-receptor blocker (ARB) might be harmful or protective in COVID-19. (A) SARS-CoV-2 anchors on transmembrane angiotensinconverting enzyme 2 (ACE2) to enter host cells. (B) The addition of an ACEi or ARB could increase ACE2 abundance and thus enhance viral entry. (C) Vasoconstrictor angiotensin II (ANG II) drives lung injury by binding to the type 1 angiotensin receptor (AT1R), causing downstream cascade of inflammation and fibrosis. (D) Diminishing production of ANG II with an ACEi or blocking ANG II-AT1R actions with an ARB enhances the generation of beneficial Ang(1-7) by ACE2 and activates the Mas receptor (MasR), which attenuates lung injury through anti-fibrotic, anti-inflammatory, and vasodilatory properties.

ACE, a homolog of ACE2, converts ANG I (angiotensin I-a decapeptide pro-hormone) into ANG II and also degrades Ang-(1-7). By increasing ANGII, it exerts many deleterious effects on the cardiopulmonary system through stimulating myocyte hypertrophy, fibroblast proliferation, and aldosterone secretion that can potentiate cardiac fibrosis and inflammation. ACE inhibitors thus have been a standard of choice in treating heart failure patients and have shown promising effects on cardiac function and survival [22]. 
Concerns have been raised that antihypertensive medications, such as ACE inhibitors (ACEIs) and angiotensin II receptor blockers (ARBs), could negatively impact the risk of SARS-CoV-2 infection and lead to severe COVID-19 owing to the role of ACE2 as the virus receptor (Figure 2). However, there are no clinical data currently available that support or reject this notion [2, 6, 23]. Research in animal models has demonstrated that ACEIs/ARBs upregulate ACE2 in heart and kidney [26, 27], raising speculations over increased susceptibility of ACEIs/ARBs consumers to SARS-CoV-2 infection or developing severe COVID-19. In contrast, blockade of angiotensin type I receptor (AT1R) in mice infected with SARSCoV, protected against ANG II-induced acute lung injury and pulmonary edema [28]. Due to a lack of sufficient evidence in humans, American Heart Association (AHA) and other expert guidelines recommend against discontinuation of RAS inhibitors in CVD patients with COVID-19. Whether ACEIs/ARBs are worsening clinical outcomes by facilitating ACE2-mediated virus entry or protecting against virusinduced cardiac and pulmonary damage through increased Ang-(1-7) and decreased ANG II, remain to be seen (Figure 2). In fact, a couple of clinical trials with ARB Losartan (NCT04335123, NCT04312009) are underway in patients with COVID-19 to determine its efficacy in mitigating lung injury and hospitalization. Trials like this, along with observational studies, are needed to assess the complex interplay of SARS-CoV-2 with the RAS network and how that will aid in adapting treatment strategies for reducing the burden of COVID-19 among CVD patients.

\section{Conclusions}

As COVID-19 pandemic evolves in real-time, so is our understanding of the disease and its causative agent SARS-CoV-2. But, we still have long ways to go in terms of finding effective clinical measures and therapeutic paradigms for the treatment of COVID-19. Though severe respiratory illness remains a significant cause of mortality, pre-existing cardiovascular conditions particularly raise the risk of developing severe disease with poor prognosis. Virus-induced lymphocytopenia and hyper inflammation could promote the development of new-onset cardiac injury in COVID-19 patients, although the mechanisms remain poorly understood. Since there are no indications of any detrimental or beneficial effects of consuming ACEIs and ARBs on the susceptibility to SARS-CoV-2 and the severity of COVID-19, their usage should not be discontinued in patients with heart failure and other CVD. As new evidence emerges from large-scale clinical studies and retrospective analyses, it would be prudent to evaluate associations of current therapies in COVID-19 clinical outcomes and adopt tailored treatment strategies that will reduce the burden of disease in cardiovascular patients.

\section{Author's Biography}

Dr Sangeeta Chakraborty is currently a postdoctoral research fellow at the National Jewish Health, Denver, Colorado, which is a leading respiratory hospital in the United States. Her current research activities are in the field of precision medicine driven translational research of chronic ailments 
with an emphasis on the matrix biology of idiopathic pulmonary fibrosis (IPF) using synthetic hydrogels which mimic the lung extracellular matrix (ECM). The study is expected to lead to the fundamental understanding of the ECM that will inform future diagnostic tools and therapeutic approaches in IPF. She received her PhD from Indian Institute of Science, Bangalore in India. Her PhD research was funded by the Council of Scientific and Industrial Research, India. Dr. Chakraborty's pioneering research on bacterial infectious diseases and molecular mechanisms of stress resistance in the setting of host-pathogen interaction has yielded a number of significant first author publications and federal grant funding. She also work as a freelance science writer and is actively engaged in bringing the latest academic research, clinical breakthroughs, and biopharma news to consumers and healthcare professionals. Her articles can be found on GeneOnline biotechnology platform (GeneOnline.news/en/). She intends to leverage her academic background and communication skills to successfully convey scientific discoveries and advancement to diverse audiences.

Dr. Chakraborty can be reached at Sangeeta1986@gmail.com.

\section{References:}

1. Centers for Disease Control and Prevention. Coronavirus Disease 2019 (COVID-19).

https://www.cdc.gov/coronavirus/2019ncov/cases-updates/cases-in-us.html

2. Madjid, Mohammad, et al. "Potential effects of coronaviruses on the cardiovascular system: a review." JAMA cardiology (2020).

DOI:10.1001/jamacardio.2020.1286

3. Zhou, Peng, et al. "A pneumonia outbreak associated with a new coronavirus of probable bat origin." nature 579.7798 (2020): 270-273. DOI: $10.1038 / \mathrm{s} 41586-$ 020-2012-7

4. Cheng, Paul, et al. "Cardiovascular Risks in Patients with COVID-19: Potential Mechanisms and Areas of Uncertainty." Current Cardiology Reports 22.5 (2020). DOI: $10.1007 / \mathrm{s} 11886-020-01293-2$

5. Wu, Chaomin, et al. "Risk factors associated with acute respiratory distress syndrome and death in patients with coronavirus disease 2019 pneumonia in Wuhan, China." JAMA internal medicine (2020).

DOI:10.1001/jamainternmed.2020.0994

6. Clerkin, Kevin J., et al. "Coronavirus disease 2019 (COVID-19) and cardiovascular

disease." Circulation (2020).

DOI: $\underline{10.1161 / C I R C U L A T I O N A H A .120 .0}$ 46941

7. Zhou, Fei, et al. "Clinical course and risk factors for mortality of adult inpatients with COVID-19 in Wuhan, China: a retrospective cohort study." The lancet (2020).

DOI:https://doi.org/10.1016/S01406736(20)30566-3

8. Wu, Zunyou, and Jennifer M. McGoogan. "Characteristics of and important lessons from the coronavirus disease 2019 (COVID-19) outbreak in China: summary of a report of 72314 cases from the Chinese Center for Disease Control and 
Prevention." Jama 323.13 (2020): 12391242. DOI:10.1001/jama.2020.2648

9. Johns Hopkins University and Medicine. Coronavirus resource center. https://coronavirus.jhu.edu/data/mortality

10. Onder, Graziano, Giovanni Rezza, and Silvio Brusaferro. "Case-fatality rate and characteristics of patients dying in relation to COVID-19 in Italy." Jama (2020). DOI:10.1001/jama.2020.4683

11. Li, Bo, et al. "Prevalence and impact of cardiovascular metabolic diseases on COVID-19 in China." Clinical Research in Cardiology (2020): 1-8. DOI: $10.1007 / \mathrm{s} 00392-020-01626-9$

12. Huang, Chaolin, et al. "Clinical features of patients infected with 2019 novel coronavirus in Wuhan, China." The lancet 395.10223 (2020): 497-506. DOI: 10.1016/S0140-6736(20)30183-5

13. Wu, Zunyou, and Jennifer M. McGoogan. "Characteristics of and important lessons from the coronavirus disease 2019 (COVID-19) outbreak in China: summary of a report of 72314 cases from the Chinese Center for Disease Control and Prevention." Jama 323.13 (2020): 12391242. DOI:10.1001/jama.2020.2648

14. Sławiński, G., and Ewa Lewicka. "What should a cardiologist know about coronavirus disease 2019?." Kardiologia Polska 78.4 (2020): 278-283. DOI: $10.33963 / \mathrm{KP} .15302$

15.Zheng, Ying-Ying, et al. "COVID-19 and the cardiovascular system." Nature Reviews Cardiology 17.5 (2020): 259260. doi: $10.1038 / \mathrm{s} 41569-020-0360-5$

16. Yang, Jing, et al. "Prevalence of comorbidities and its effects in patients infected with SARS-CoV-2: a systematic review and meta-analysis." International Journal of Infectious Diseases 94 (2020): 91-95. DOI: 10.1016/j.ijid.2020.03.017

17.Guan, Wei-jie, et al. "Clinical characteristics of coronavirus disease 2019 in China." New England journal of medicine 382.18 (2020): 1708-1720. DOI: $10.1056 /$ NEJMoa2002032

18. Babapoor-Farrokhran, Savalan, et al. "Myocardial injury and COVID-19: Possible mechanisms." Life Sciences (2020): 117723. DOI: $10.1016 / \mathrm{j} . \mathrm{Ifs} .2020 .117723$

19.Wang, Dawei, et al. "Clinical characteristics of 138 hospitalized patients with 2019 novel coronavirusinfected pneumonia in Wuhan, China." Jama 323.11 (2020): 1061-1069. DOI: 10.1001/jama.2020.1585

20.Guo, Tao, et al. "Cardiovascular implications of fatal outcomes of patients with coronavirus disease 2019 (COVID19)." JAMA cardiology (2020). DOI:10.1001/jamacardio.2020.1017

21. Adão, Rui, and Tomasz J Guzik. "Inside the heart of COVID-19." Cardiovascular research vol. 116,6 (2020): e59-e61. DOI: $\underline{10.1093 / \text { cvr/cvaa086 }}$

22. South, Andrew M., Debra I. Diz, and Mark C. Chappell. "COVID-19, ACE2, and the cardiovascular consequences." American Journal of Physiology-Heart and Circulatory Physiology 318.5 (2020): H1084-H1090.

DOI: 10.1152/ajpheart.00217.2020

23. Gonzalez-Jaramillo, Nathalia et al. "The double burden of disease of COVID-19 in cardiovascular patients: overlapping conditions could lead to overlapping treatments." European journal of 
epidemiology vol. 35, 4 (2020): 335-337. DOl.org/10.1007/s 10654-020-00628-1

24. Imai, Yumiko et al. "Angiotensinconverting enzyme 2 protects from severe acute lung failure." Nature vol. 436,7047 (2005). DOI: $\underline{10.1038 / \text { nature03712 }}$

25. Patel, Ankit B., and Ashish Verma. "Nasal ACE2 Levels and COVID-19 in Children." JAMA.

DOI:10.1001/jama.2020.8946

26. Ishiyama, Yuichiro, et al. "Upregulation of angiotensin-converting enzyme 2 after myocardial infarction by blockade of angiotensin

receptors." Hypertension 43.5 (2004):
970-976.

DOI:10.1161/01.HYP.0000124667.34652 .1a

27. South, Andrew M., et al. "Controversies of renin-angiotensin system inhibition during the COVID-19 pandemic." Nature Reviews Nephrology (2020): 1-3. DOI:https://doi.org/10.1038/s41581-020$\underline{0279-4}$

28. Kuba, Keiji, et al. "A crucial role of angiotensin converting enzyme 2 (ACE2) in SARS coronavirus-induced lung injury." Nature medicine 11.8 (2005): 875-879.

DOI: 


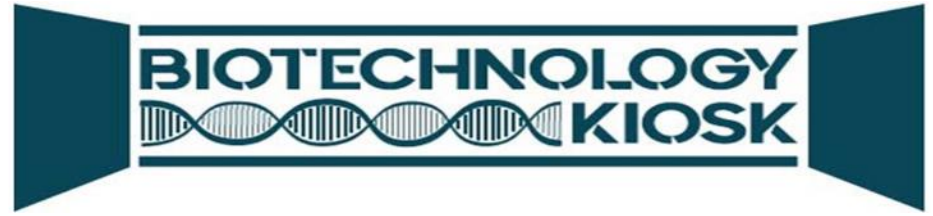 \\ Biotechnology Advances around the World Editor's Picks}

Every issue of Biotechnology Kiosk presents select latest research news picked by the editorsin-chief on significant research breakthroughs in different areas of biotechnology around the world. The aim is to promote further R\&D in all of these cutting edge areas of biotechnology. The editors have compiled and included the following innovations and breakthroughs to highlight the recent biotechnology advances.

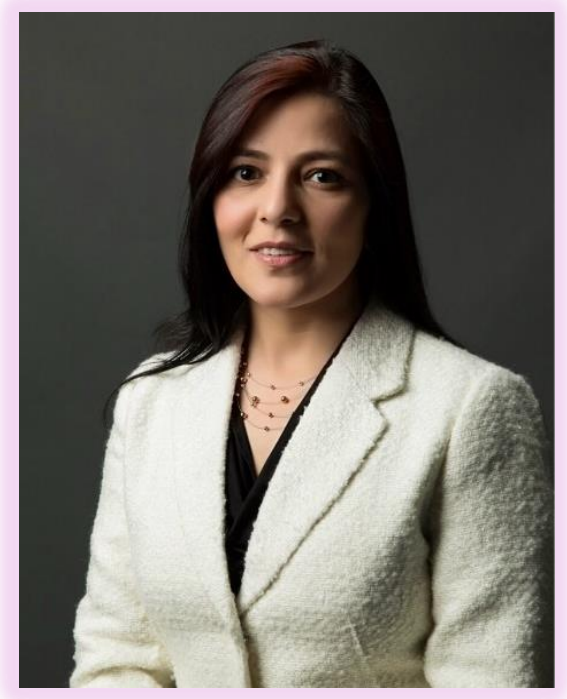

Dr. Megha Agrawal

Co Editor-in-Chief

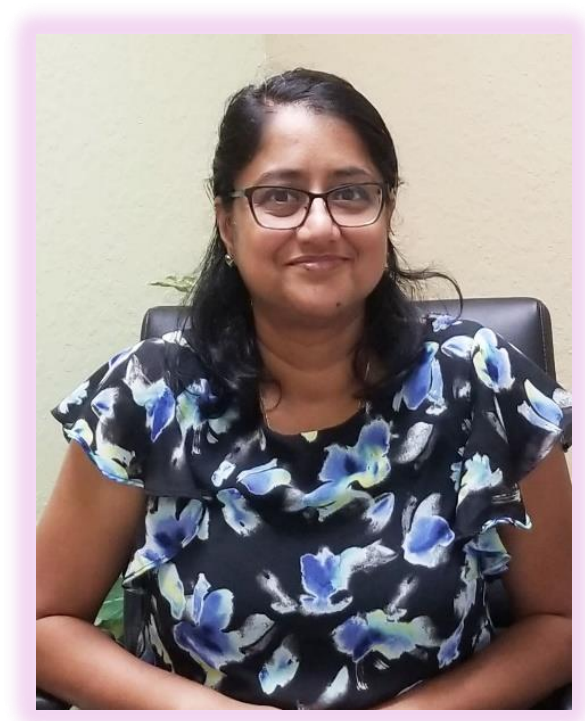

Dr. Shyamasri Biswas
Co Editor-in-Chief 


\section{Cancer Biology}

\section{An adenovirus to target and kill cancer cells}

Previous research studies showed the potential of genetically modifying adenoviruses to kill cancers. These adenoviruses are known to replicate inside cancer cells and kill them when injected inside the cells. This has prompted clinical trials of these viruses to exploit their potential.

This research has made further advances. Scientists in Japan are now trying to design more efficient viruses. This aim is to create these viruses with additional functionality that are better able to target cancer cells while not harming normal cells. To this end, researchers employed special RNAstabilizing elements in an adenovirus that specifically replicates inside and kills cancer cells. Their research were published in the journal Cancers (Conditionally Replicative Adenovirus Controlled by the Stabilization System of AU-Rich Elements Containing mRNA, Cancers, 2020; 12 (5): 1205 DOI: 10.3390/cancers12051205).
In this study, researchers made two new adenoviruses to specifically target cancer cells. They employed 'adenylate-uridylaterich elements' (AREs). These elements have been studied and they are known as signals in RNA molecules that enhance the rapid decay of messenger RNAs (mRNAs) in human cells. Genes that are required for cell growth and proliferation are known to have AREs. Researchers showed that AREcontaining mRNAs can become temporarily stabilized under certain stress conditions. This allowed the maintenance of some necessary cell processes. Further, AREmRNAs were also shown to be stabilized in cancer cells. This process supported their continuous proliferation.

This study paves the ARE-mRNA stability to be applied for diseases other than cancer. Researchers envisioned that the engineered adenoviruses could have potential for treating diseases that are linked to inflammations, viral infection, hypoxia, and ultraviolet irradiation.

\section{Microorganisms and Bacteria}

\section{Producing metal using microorganisms}

It has been observed that microorganisms such as fungi and bacteria and also plants generate a wide range of chemical substances that are called secondary metabolites. These metabolites are created as a response to the environmental conditions. These chemical substances produce metal-binding molecules called chelators. One of the industrially-important chelators is the iron-binding siderophores. These iron-binding molecules are essential for many metabolic processes due to the presence of iron, which is an essential component of many enzymes and signalling pathways.

This can be understood by the example of pathogenic bacteria that use siderophores to extract iron from their host for their metabolism. The host that might become iron 
deficient could be compensated by the siderophores that can also used by bacteria living in soil. This makes the process accessible to iron that allows to gain an advantage over other organisms in the same habitat. There are other various metals and metalloids including zinc, vanadium, molybdenum or even uranium oxides that are found in such chelators.

In a recent review article, researchers in Germany described the different natural chelators and their ability to bind metals and metalloids. This study explored current and potential future applications of metal-binding substances made of bacteria and other microorganisms. They published their article in Natural Product Reports (Metal binding ability of microbial natural metal chelators and potential applications, Natural Product Reports, 2020; DOI: 10.1039/C9NP00058E).

Researchers discussed that such chelators may have many potential applications that include floors remediation, selectively extract or separate raw materials, or even in biosensorics or medicine. Further, they envisioned applications of siderophores in medical applications as well by leveraging them to treat iron overload in the body known as iron storage disorder.

\section{Regenerative Medicine}

Rejuvenation of fibroblasts by mechanical reprogramming

Fibroblasts are the most common connective tissue cells and considered vital constituents of the connective tissue in humans. These constituents provide the required mechanical strength and they maintain tissue homeostasis by promoting extracellular matrix (ECM) remodeling. They are also known to produce the structural framework for animal tissues that is used to synthesize the ECM and the important collagen that play a crucial role in wound healing. However, over the course of the normal cellular aging process, fibroblasts gradually lose their ability to contract that leads to reduced connective-tissue stiffness. To overcome this challenge, a promising therapeutic avenue could be functional rejuvenation of connective tissue by the reprogrammed fibroblast replacement.
Researchers in Singapore have addressed this issue in a study from that has shown that these fibroblasts can be rejuvenated, or redifferentiated, by being geometrically confined on micropatterns. They showed these rejuvenated cells with reduced DNA damage, and enhanced cytoskeletal gene expression. These results were recently published in the Proceedings of the National Academy of Sciences on 29 April 2020 (Fibroblast rejuvenation by mechanical reprogramming and redifferentiation, Proceedings of the National Academy of Sciences, 2020; 117 (19): 10131 DOI: 10.1073/pnas.1911497117).

In this study, researchers embedded the partially reprogrammed spheroids in collagen-I matrices of varying densities that mimicked different three-dimensional (3D) tissue constraints. Subsequently, these spheroids were observed to regain their 
fibroblastic properties in response to such matrix constraints that sprouted and resulted in 3D connective-tissue networks. Further, these differentiated fibroblasts were shown to exhibit reduced DNA damage, enhanced cytoskeletal gene expression, and actomyosin contractility. Also, it was demonstrated that the rejuvenated fibroblasts enhanced matrix protein (fibronectin and laminin) deposition and collagen remodeling compared to the parental fibroblast tissue network. These results highlighted efficient fibroblast rejuvenation through laterally confined reprogramming and thus, may have important implications in regenerative medicine.

\section{Plant and Agriculture Biotechnology}

Genetic engineering of brome mosaic plant virus for saving wheat and other crops

Brome Mosaic virus is a plant virus and known to primarily affect grasses such as wheat and barley, and also soybeans as well. The genetic material of this virus has been shown to be divided into three particles. Studies have shown that inside of each of the particles comprises a strand of RNA, which is the genetic material that is believed to control the production of proteins. Subsequently different detrimental effects are caused by these proteins on the plants that include stunted growth, lesions, and ultimately death of infected host plants.

Despite research advances made in this area, up until now, researchers couldn't fully understand how they work together to initiate an infection that destroys food crops due to the lack of a more definitive picture of the differences between these particles. Researchers in the USA addressed this issue in a recent study using a genetic engineering technique that disabled the pathogenic aspects of the virus and infused the viral genes with a host plant. Their research was published in the journal PNAS (Genome organization and interaction with capsid protein in a multipartite RNA virus, Proceedings of the National Academy of Sciences, 2020; 201915078 DOI: 10.1073/pnas.1915078117).

Researchers used the bacterium and inserted its genome into the plant's cells following the similar strategy of HIV insertion into human cells. By following this procedure, they were then able to isolate the viral particles in the plants and determine their structure using electron microscopes and computer-aided technology. They subsequently mapped one of the particles that revealed that the first two particles were more stable than the third. With these results, researchers envisioned that it would be possible to manipulate the release of RNA released into the plants. Their study shows the possibility of making the third particle more stable that would allow to manipulate release of RNA to delay the infection in crops.

\section{Compiled and Edited by}

Dr. Megha Agrawal \& Dr. Shyamasri

Biswas

Co Editors-in-Chief 


\section{\begin{tabular}{l} 
BOTECHNOIOGE \\
BODOMKKIOSK \\
\hline
\end{tabular}}

\section{Biotech and Pharma Industry Roundup}

\section{Eli Lilly's Tauvid imaging agent} approved by FDA to diagnose Alzheimer's disease

Two abnormal proteins amyloid and tau are known to be associated with Alzheimer's disease. Eli Lilly recently announced the approval its imaging agent, Tauvid the U.S. Food and Drug Administration (FDA). Tauvid is a radioactive diagnostic agent that is used in positron emission tomography (PET) imaging in Alzheimer's patients. It is to be noted that Tauvid is not a treatment but is used in diagnosis, and the first and only approved diagnostic agent for imaging tau neurofibrillary tangles (NFTs) in the brain. Tauvid was developed by Avid Radiopharmaceuticals, a wholly owned subsidiary of Eli Lilly [Source: https://www.biospace.com/].

\section{Menlo Therapeutics' ZILXI ${ }^{\mathrm{TM}}$} (minocycline) topical foam, $1.5 \%$ receives FDA approval for the treatment of inflammatory lesions of rosacea in adults

Rosacea is known as a diverse skin condition. The most commonly symptoms of rosacea are deep facial redness, spider veins (telangiectasia) and acne-like inflammatory lesions (papules and pustules). Menlo Therapeutics Inc., which is a specialty pharmaceutical company focused on therapies to address unmet needs in dermatology recently announced the approval of ZILXI ${ }^{\mathrm{TM}}$ (minocycline) topical foam, $1.5 \%$ by the FDA. ZILXI TM is used for the treatment of inflammatory lesions of rosacea in adults. ZILXI was developed as FMX103 by Menlo's wholly-owned subsidiary Foamix Pharmaceuticals Ltd. ("Foamix"), is considered the first minocycline product of any kind to be approved by the FDA for use in rosacea. This FDA approval is considered very encouraging news for clinicians and patients seeking options for this difficult to treat skin disorder [Source: https://www.biospace.com/].

\section{Eagle Pharma to test MH Drug Ryanodex against COVID-19}

Eagle Pharmaceutical recently demonstrated in a controlled laboratory test, the efficacy of their malignant hyperthermia treatment Ryanodex (dantrolene sodium) that inhibited the growth of SARS-CoV-2, the virus causing the COVID-19 pandemic. In the in-vitro tests, Ryanodex showed antiviral activity and a lack of cytotoxicity. With this success, the company now hopes to launch a clinical trial testing the efficacy of the drug in patients in the near future [Source: https://www.biospace.com/]. 


\section{Meningococcal Vaccines expected to touch US\$ 9 billion by 2026}

Bacteria Neisseria meningitides causes meningococcal disease. This disease occurs throughout the world especially in the developing world. Typical symptoms include inflammation in the membranes of the brain and spinal cord which is characteristic of meningitis. The meningococcal vaccines market is pretty vast with a lot of potential to make its footprints even larger in the future that is expected to reach $\$ 9$ billion by 2026 . This vaccine exists in the market and doing a great business due to its utility and need for preventing the incidence of this deadly disease https://www.biospace.com/].

[Source:

\section{Novartis partners with Massachusetts Eye \& Ear hospital to manufacture coronavirus vaccine}

In a recent announcement, Novartis' AveXis division said that it will manufacture a coronavirus vaccine partnering with Massachusetts Eye and Ear Hospital. This partnership is supposed to pave the way for the academic institution to take advantage of the technology that has delivered the gene therapy Zolgensma to spinal muscular atrophy patients. It is expected that AveXis will begin manufacturing the vaccine as soon as possible with the partnering hospital expecting to begin a Phase 1 trial in humans in the second half of 2020 [Source: https://www.biopharmadive.com/).

\section{AstraZeneca to study leukemia drug as COVID-19 treatment}

In an effort to repurpose existing therapies for the fast-spreading disease, AstraZeneca will go into clinical study for a leukemia drug that could help calm the overactive immune response seen in some patients with COVID19

https://www.biopharmadive.com/)].

[Source:

\section{Large scale phase 2 and phase 3 trials are underway for coronavirus vaccine}

Late-stage clinical trials are underway involving tens of thousands of people for coronavirus vaccine development. Phase 2 and phase 3 trials are being prepared in the U.S., Europe and also other regions due to the fact that the pandemic's heaviest impact has begun to shift to the Southern Hemisphere. This large scale clinical trials correspond to an important transition from an early safety studies of coronavirus vaccines that involved several dozens to hundreds of healthy volunteers. But considering the uniqueness of this global pandemic that will require safety and effectiveness of a treatment to be potentially given to tens of millions of people globally, massive trials spanning many countries are required. To this end, a phase 2 study of a vaccine being developed by the University of Oxford and AstraZeneca aims to recruit 10,260 adults and children in the U.K. In addition, the British drug maker is planning a phase 3 trial in the U.S. involving about 30,000 people [Source: https://www.biopharmadive.com/)]. 
Investors pouring cash to counter cancer genes

It is known for years that a family of genes that, when mutated, turn regular cells cancerous. There has been recent progress in developing drugs that can effectively silence those genes. This has fueled investor optimism in companies going after these genes that are known as RAS. The most recent example of investments comes from a California based biotech company named Revolution Medicines that has raised \$238 million through an initial public offering. RAS genes are known to make special proteins that regulate how a cell grows, specializes and divides. One of these proteins, K-ras, has drawn a particularly large amount of attention for R\&D. and Amgen, the country's largest biotech has released data showing an experimental drug appeared to stabilize disease in a small group of lung cancer patients with KRAS gene mutations. This has further fueled extensive $R \& D$ in this area [Source: https://www.biopharmadive.com/)].

\section{Roche presents biomarker for checkpoint block blockade}

Researchers at Swiss pharma giant Roche AG have demonstrated a subpopulation of dendritic cells that determines how cancer patients respond to immune checkpoint inhibitors [Source: https://europeanbiotechnology.com/].

\section{Dutch Biotech Company HALIX B.V. to produce vaccines against COVID-} 19

Dutch CDMO HALIX B.V. has entered in a research consortium coordinated by the University of Oxford, to provide GMPcompliant production of Vaccitech Ltd's COVID-19 vaccine (ChAdOx1 nCoV-19) targeting the viral spike protein [Source: https://european-biotechnology.com/].

\section{SARS-CoV2 antibody tests might not be suitable for individual diagnosis}

An international research consortium in Europe has provided data that demonstrate that SARS-CoV-2 antibody tests are only useful for population-based analyses and might not work for individual diagnosis. [Source: https://europeanbiotechnology.com/]. 


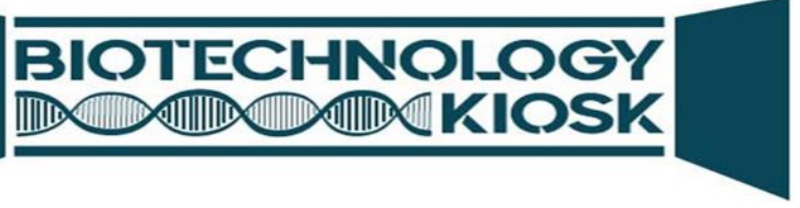

For all production related questions or sending your ads, please email or call our production department: E-mail: sales@biotechkiosk.com; Phone: 386-518-9411.

THANK YOU! 